\title{
Loss of the Notch effector RBPJ promotes tumorigenesis
}

\author{
Iva Kulic, ${ }^{1,4}$ Gordon Robertson, ${ }^{1}$ Linda Chang, ${ }^{1}$ Jennifer H.E. Baker, ${ }^{2}$ \\ William W. Lockwood, ${ }^{2}$ Winnie Mok, ${ }^{1}$ Megan Fuller, ${ }^{1}$ Michèle Fournier, ${ }^{1}$ \\ Nelson Wong, ${ }^{1}$ Vennie Chou, ${ }^{3}$ Mark D. Robinson, ${ }^{6,7}$ Hye-Jung Chun, ${ }^{1}$ \\ Blake Gilks, ${ }^{5}$ Bettina Kempkes, ${ }^{8}$ Thomas A. Thomson, ${ }^{3}$ Martin Hirst, ${ }^{1}$ \\ Andrew I. Minchinton, ${ }^{2}$ Wan L. Lam, ${ }^{2}$ Steven Jones, ${ }^{1}$ Marco Marra, ${ }^{1}$ \\ and Aly Karsan ${ }^{1,3,4,5}$
}

${ }^{1}$ Genome Sciences Centre, ${ }^{2}$ Integrative Oncology Department, and ${ }^{3}$ Department of Pathology and Laboratory Medicine, British Columbia Cancer Agency, Vancouver, British Columbia V5Z 1L3, Canada

${ }^{4}$ Experimental Medicine Program and ${ }^{5}$ Department of Pathology and Laboratory Medicine, University of British Columbia, Vancouver V6T 2B5, British Columbia, Canada

${ }^{6}$ Institute of Molecular Life Sciences and 7SIB Swiss Institute of Bioinformatics, University of Zurich, CH-8057 Zurich, Switzerland ${ }^{8}$ Department of Gene Vectors, Helmholtz Zentrum München, German Research Center for Environmental Health,

81377 Munich, Germany

Aberrant Notch activity is oncogenic in several malignancies, but it is unclear how expression or function of downstream elements in the Notch pathway affects tumor growth. Transcriptional regulation by Notch is dependent on interaction with the DNA-binding transcriptional repressor, RBPJ, and consequent derepression or activation of associated gene promoters. We show here that $R B P J$ is frequently depleted in human tumors. Depletion of RBPJ in human cancer cell lines xenografted into immunodeficient mice resulted in activation of canonical Notch target genes, and accelerated tumor growth secondary to reduced cell death. Global analysis of activated regions of the genome, as defined by differential acetylation of histone $\mathrm{H} 4$ ( $\mathrm{H} 4 \mathrm{ac})$, revealed that the cell death pathway was significantly dysregulated in RBPJdepleted tumors. Analysis of transcription factor binding data identified several transcriptional activators that bind promoters with differential H4ac in RBPJ-depleted cells.

Functional studies demonstrated that NF- $\mathrm{KB}$ and MYC were essential for survival of RBPJdepleted cells. Thus, loss of RBPJ derepresses target gene promoters, allowing Notchindependent activation by alternate transcription factors that promote tumorigenesis.

CORRESPONDENCE

Aly Karsan:

akarsan@bcgsc.ca

Abbreviations used: ChIP, chromatin immunoprecipitation; DAPT, $N-[N-(3,5-$ difluorophenacetyl)-L-alanyl]-S-phenylglycine t-butyl ester; EMSA, electrophoretic mobility shift assay; $\mathrm{H} 3 \mathrm{~K} 4 \mathrm{me} 3$, histone $\mathrm{H} 3$ lysine 4 tri-methylation; H3K9me3, histone H3 lysine9 tri-methylation; H4ac, acetylated histone H4; HD, homozygous deletion; mt, mutant; sh, short hairpin; TSS, transcription start site; TUNEL, terminal deoxynucleotidyl transferase dUTP nick end labeling.
Notch signaling is directed through RBPJ, the DNA-binding component of the pathway (Kovall and Hendrickson, 2004; Borggrefe and Oswald, 2009). RBPJ is ubiquitously expressed and acts as a transcriptional repressor in the absence of active Notch (Hamaguchi et al., 1992; Bray, 2006). Binding of active Notch to RBPJ results in expulsion of a histone deacetylase-containing corepressor complex and recruitment of histone acetyltransferases to the Notch-RBPJ ternary complex to facilitate chromatin remodeling and transcriptional activation (Borggrefe and Oswald, 2009). Increased expression of Notch1 or its ligand Jagged1 is associated with poor survival in breast and other cancers (Reedijk et al., 2005; Koch and Radtke, 2007). To evaluate the relevance of RBPJ in tumor promotion, we examined RBPJ mRNA and protein levels in primary human cancers and modeled RBPJ depletion in tumor xenograft studies.

\section{RESULTS}

RBPJ is frequently lost in human cancers

To determine whether altered RBPJ expression is associated with oncogenesis, we performed immunohistochemical staining of 264 human breast carcinoma cases. Immunostaining revealed lack of RBPJ protein in 15\% (40/264) of cases, whereas nonmalignant breast tissue showed high levels of epithelial expression (Fig. 1 A). RBPJ loss did not correlate with hormone receptor or human epidermal growth factor receptor 2 status

2015 Kulic et al. This article is distributed under the terms of an AttributionNoncommercial-Share Alike-No Mirror Sites license for the first six months after the publication date (see http://www.rupress.org/terms). After six months it is available under a Creative Commons License (Attribution-NoncommercialShare Alike 3.0 Unported license, as described at http://creativecommons.org/ licenses/by-nc-sa/3.0/). 
(unpublished data). Examination of microarray data from independent studies confirmed significantly reduced RBPJ mRNA expression in breast cancers (Fig. 1 B; Yu et al., 2008). Using TCGA data (Network, 2012), we evaluated RBPJ copy loss and mRNA expression in invasive breast cancers. Genomic loss of RBPJ occurred in 33\% (277/828) of cases, and this coincided with significantly reduced transcript levels (Fig. 1 C). Cases either with RBPJ homozygous deletion (HD; $n=7$ ) and loss $(n=270)$ showed the lowest RBPJ expression (Fig. 1 D).
Analysis of microarray data from a study in which tumors were classified by grade showed that RBPJ expression was preferentially reduced in higher-grade breast cancers (Fig. $1 \mathrm{E}$; Ginestier et al., 2006), suggesting that reduced RBPJ expression may be associated with more aggressive tumors. Of interest, a significant negative correlation between expression of $R B P J$ and its canonical target gene, HEY2, was observed in the grade 3 subset $\left(n=39, \mathrm{R}^{2}=0.2\right.$, Pearson $\left.\mathrm{P}=0.003\right)$. A separate invasive lobular breast carcinoma dataset also showed
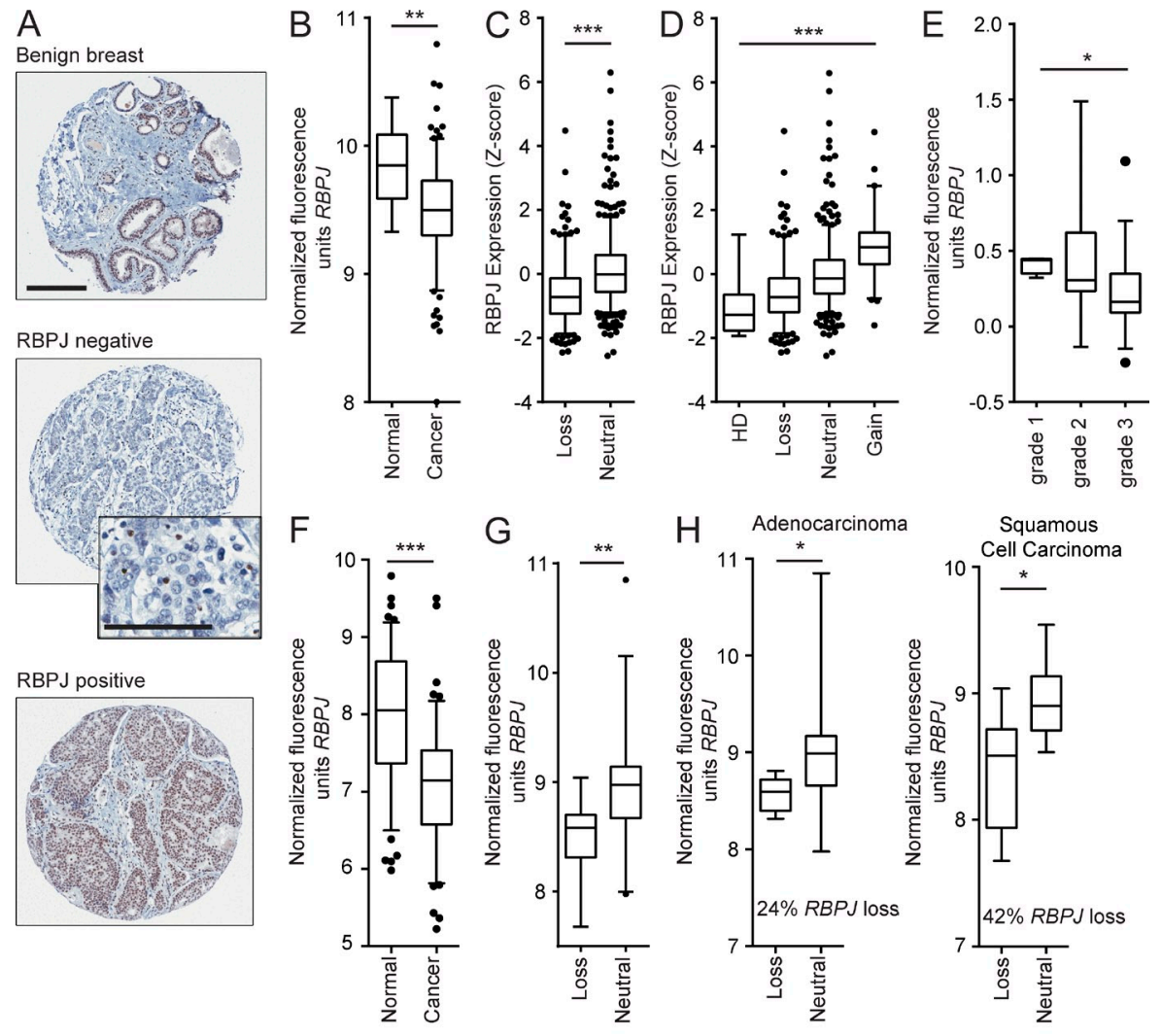

I Adenocarcinoma

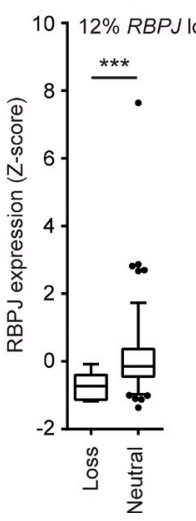

Squamous Cell Carcinoma

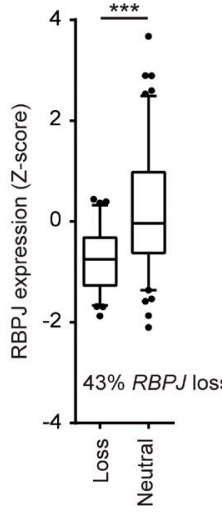

$J$

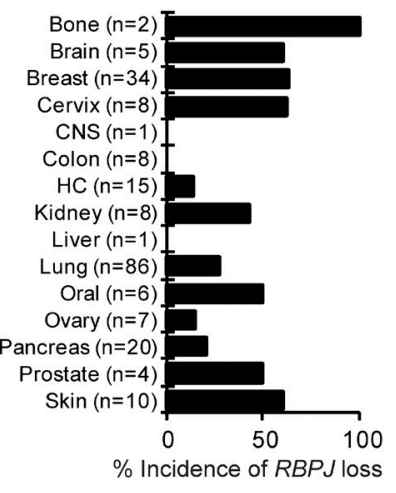

Figure 1. $R B P J$ is frequently lost in human cancers. (A) Examples of RBPJ immunohistochemical staining in benign breast tissue $(n=8)$ and breast cancer tissue microarray cores (RBPJ negative, $n=40$; RBPJ positive, $n=224 ;$ bar, $200 \mu \mathrm{m}$ ). High power inset (bar = $100 \mu \mathrm{m}$ ) of the RBPJ-negative tumor core shows positive staining in internal control cells in the tumor microenvironment. (B) RBPJ mRNA expression in breast tumors $(n=183)$ and adjacent normal breast tissue ( $n=13 ; \mathrm{Yu}$ et al., 2008). (C) Analysis of RBPJ expression and RBPJ genomic copy loss ( $n=277$ ) versus no loss (neutral, $n=551$ ) in invasive breast cancers (TCGA data). (D) Data from C plotted by $R B P J$ copy number status; $\mathrm{HD}(n=7)$, loss ( $n=270)$, neutral $(n=489)$, and amplification (gain, $n=62$ ). $P<0.0001$ by Kruskal-Wallis followed by Dunn's multiple comparisons post-test showed significant differences in all comparisons except between the HD versus loss group. (E) RBPJ mRNA expression in human breast cancers stratified by tumor grade; grade $1(n=4)$, grade $2(n=12)$, and grade 3 ( $n=39$; Ginestier et al., 2006). (F) RBPJ mRNA expression in normal bronchial epithelium collected from healthy individuals $(n=67)$ versus non-small cell lung carcinoma $(n=111$; Bild et al., 2006; Lockwood et al., 2010).

$(G)$ Analysis of lung cancers of mixed type with RBPJ genomic copy loss ( $n=14$ ) versus no loss $(n=30)$ with paired mRNA expression and aCGH data (Lockwood et al., 2008, 2010). $(H)$ Analysis of lung cancers from $G$ broken down into tumor subtypes; adenocarcinoma RBPJ genomic loss $(n=6)$ versus no loss $(n=19)$; squamous cell carcinoma RBPJ genomic loss $(n=8)$ versus no loss ( $n=11$; Lockwood et al., 2008, 2010). (I) Analysis of RBPJ mRNA expression and genomic copy number in TCGA lung cancer adenocarcinomas (RBPJ genomic loss [ $n=15]$ versus no loss [ $n=114]$ ) and squamous cell carcinomas (RBPJ genomic loss $[n=77]$ versus no loss [ $n=101]$; Cerami et al., 2012; Lockwood et al., 2012; Gao et al., 2013). (J) RBPJ copy number alteration evaluated using aCGH across a panel of 215 cancer cell lines (CNS: central nervous system, HC: hematopoietic cell lines). At least one allele of RBPJ is lost at an overall frequency of 35\% (also see Table S1). P-values were obtained using a Mann-Whitney test for B, C, and F-I and a Kruskal-Wallis one-way analysis of variance test for $D$ and $E$. Data are presented as box-and-whisker plots. The bottom and top bars of the whisker indicate the 5th and 95th percentiles respectively with points outside the whiskers shown as individual dots. ${ }^{*}, \mathrm{P}<0.05,{ }^{* *}, \mathrm{P}<0.005,{ }^{* *}, \mathrm{P}<0.0001$. 

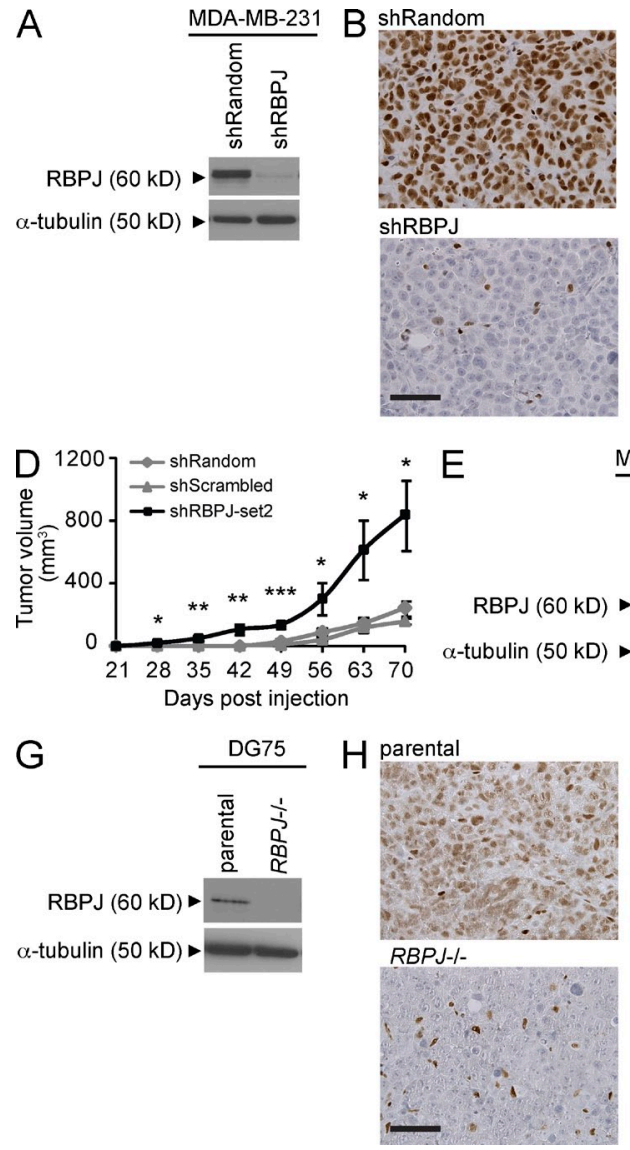
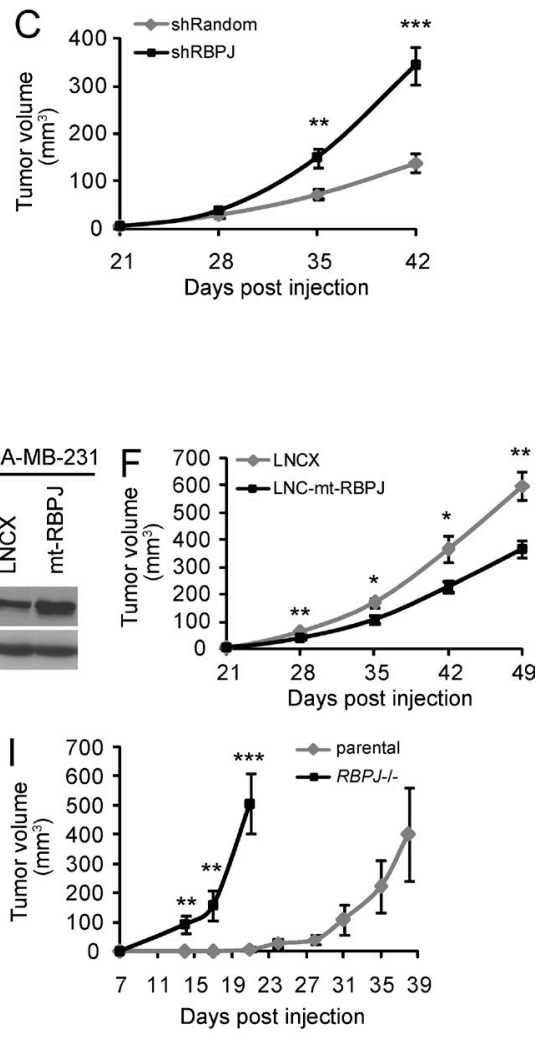

Figure 2. $R B P J$ deficiency promotes tumor growth. (A and B) Analysis of MDAMB-231 tumors transduced with an RBPJ knockdown construct (shRBPJ) or a nonspecific shRNA (shRandom). (A) Immunoblot of endpoint tumor lysates. (B) RBPJ immunohistochemistry (bar, $50 \mu \mathrm{m})$. (C) MDA-MB-231 tumor growth after expression of shRBPJ $(n=16)$ compared with shRandom control ( $n=22)$. (D) Depletion of RBPJ in MDA-MB231 tumors using an alternate RBPJ targeting construct (set2) and a different silencing control (shScrambled; $n=4$ for each group). (E) Immunoblot analysis of endpoint MDAMB-231 tumor lysates transduced with LNCX empty vector or an mt RBPJ construct (LNCmt-RBPJ). (F) MDA-MB-231 tumor growth after expression of LNC-mt-RBPJ compared with the LNCX control ( $n=8$ for each group). ( $G$ and $\mathrm{H}$ ) Analysis of parental or RBPJ-1DG75 Burkitt lymphoma tumors. (G) Immunoblot of endpoint tumor lysates. (H) RBPJ immunohistochemistry (bar, $50 \mu \mathrm{m}$ ). (I) DG75

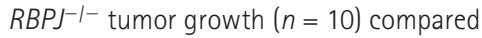
with parental DG75 tumors $(n=9)$. For $C_{1} F_{\text {, }}$ and $\mathrm{I}, \mathrm{p}$-values were obtained using a MannWhitney test and for $\mathrm{D}$ a one-way ANOVA. Data are presented as mean \pm SEM. ${ }^{*}, \mathrm{P}<0.05$; ${ }^{* *}, \mathrm{P}<0.005 ;{ }^{* * *}, \mathrm{P} \leq 0.0005$. a negative correlation between RBPJ and HEY2 mRNA expression $\left(n=18, \mathrm{R}^{2}=0.4\right.$, Pearson $\mathrm{P}=0.005$; Rhodes et al., 2004; Zhao et al., 2004).

We also evaluated RBPJ in non-small cell lung cancers $(n=$ 111) compared with normal bronchial epithelium from healthy individuals $(n=67)$ and observed reduced expression in tumors (Fig. 1 F; Bild et al., 2006; Lockwood et al., 2010). Genomic loss of at least one copy of RBPJ was associated with significantly reduced transcript levels in primary lung tumors (Fig. 1 G; Lockwood et al., 2008; Lockwood et al., 2010). Classification of tumors in Fig. $1 \mathrm{G}$ into subtypes showed that RBPJ copy number loss occurred more frequently in lung squamous cell carcinomas compared with adenocarcinomas, consistent with our analysis of TCGA lung tumor data (Fig. 1, H and I; Cerami et al., 2012; Lockwood et al., 2012; Gao et al., 2013).

Examination of 215 different tumor cell lines representing 15 tumor types showed that deletion of at least one copy of RBPJ occurred in 35\% of all cell lines (Fig. $1 \mathrm{~J}$ and Table S1). Oncomine analysis of additional cancer datasets revealed significant RBPJ underexpression in tumor tissue relative to matched normal tissue, including colon, bladder, ovarian, and gastric cancers (Rhodes et al., 2004; unpublished data). In contrast to NOTCH1, our analysis supports that RBPJ expression is reduced in several tumor types.

\section{RBPJ deficiency promotes tumor growth}

To determine whether RBPJ depletion promotes tumor progression, we knocked down RBPJ in MDA-MB-231 human breast cancer cells and subcutaneously implanted the cells into immunodeficient mice (Fig. 2, A and B). Stable RBPJ knockdown using either of two independent short hairpin (sh) RNAs significantly increased MDA-MB-231 tumor growth compared with two independent control cell lines expressing distinct nonspecific shRNAs (Fig. 2, C and D). In contrast, expression of a mutant (mt)-RBPJ (MacKenzie et al., 2004), which blocks Notch-driven transcription but permits endogenous RBPJ to bind DNA and retain repression, inhibited MDA-MB-231 tumor growth similar to direct blockade of Notch (Fig. 2, E and F; Leong et al., 2007). Before tumor seeding, the $m t-R B P J$ transcript was expressed 5.5-fold above vector control cells but only 3.5-fold in excised endpoint tumors (unpublished data). Modest overexpression of mt-RBPJ protein in endpoint tumors (Fig. 2 E) is likely due to preferential outgrowth of cells not expressing the construct. We confirmed increased tumor growth consequent to RBPJ loss in a Burkitt lymphoma cell line, DG75, in which RBPJ has been genetically ablated (Fig. 2, G-I; Maier et al., 2005). Thus, two independent methods demonstrate that RBPJ depletion promotes tumor growth in various malignancies. 
A
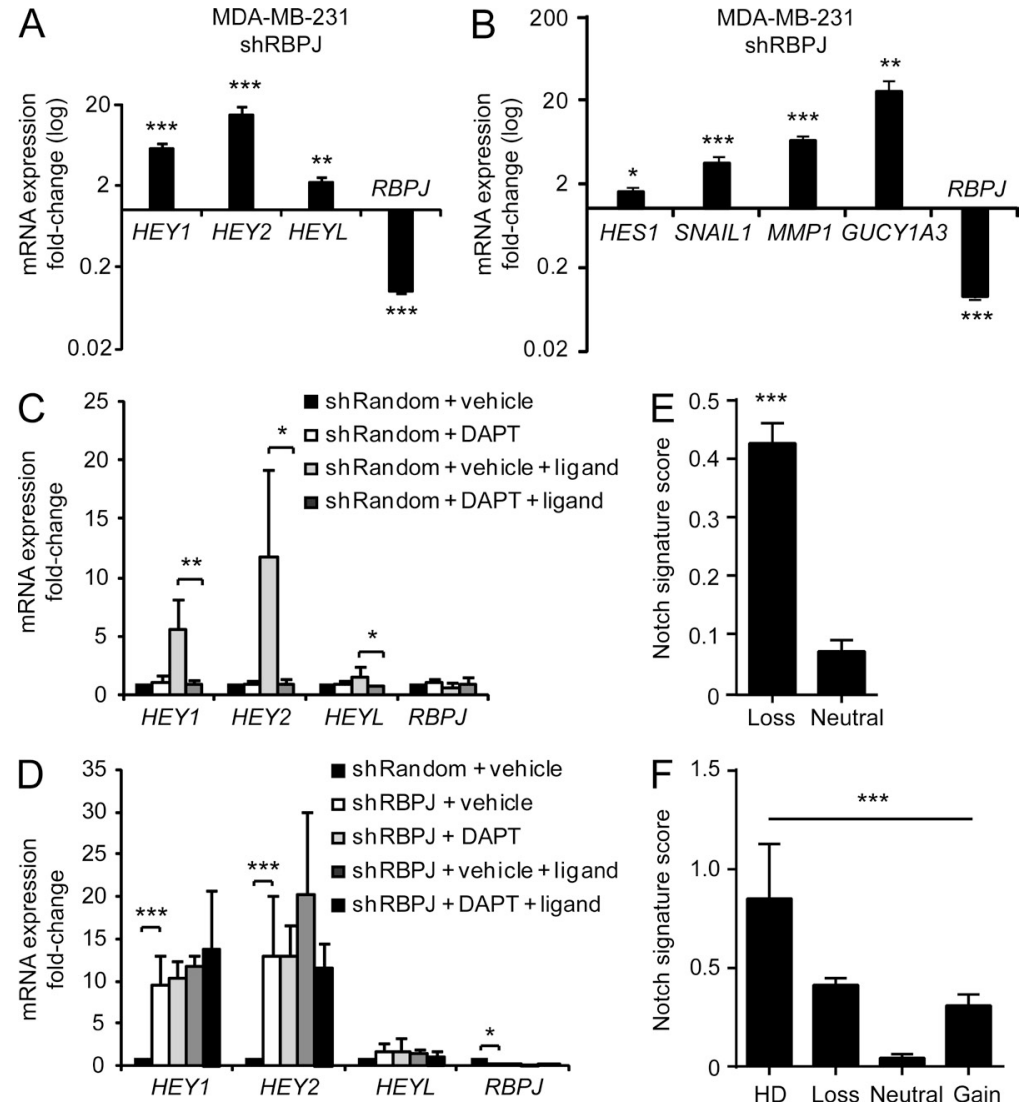
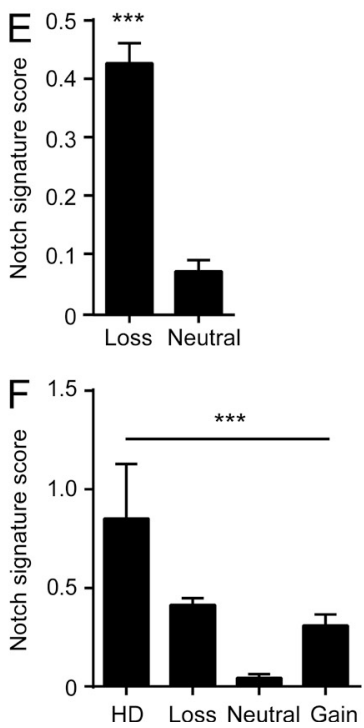

Figure 3. $R B P J$ depletion induces Notch target genes in a Notch-independent fashion. ( $A$ and $B$ ) Gene expression analysis of endpoint MDA-MB-231 tumors with data expressed as fold-change relative to the shRandom control, which corresponds to 1 on the y-axis. (A) Induction of HEY genes in shRBPJ tumors ( $n=15,90 \%$ RBPJ knockdown) compared with shRandom tumors $(n=14)$. (B) Induction of HES1, SNAIL 1, MMP1, and GUCY1A3 in shRBPJ tumors ( $n \geq 8,>90 \%$ RBPJ knockdown) compared with shRandom tumors ( $n \geq 8)$. ( $C$ and $D)$ Endogenous Notch signaling was induced in MDAMB-231 cells by Jagged 1 ligand stimulation, followed by gene expression analysis $(n=5)$. Data are expressed as fold change relative to shRandom + vehicle (DMSO). (C) HEY induction in shRandom cells is abolished by blocking Notch activation using $10 \mu \mathrm{M}$ DAPT. (D) DAPT treatment on the magnitude of $H E Y$ gene induction in shRBPJ cells ( $\geq 80 \%$ RBPJ knockdown). (E) Mean Notch signature score in a total of 828 invasive TCGA breast cancers comparing the subset with RBPJ loss ( $n=277$ ) to those without (neutral, $n=551$ ). The Notch gene signature identified in T-ALL cell lines (Margolin et al., 2009) was used to assign a score. (F) Data from E plotted by RBPJ copy number status; $\mathrm{HD}(n=7)$, loss $(n=270)$, neutral $(n=489)$, and amplification (gain, $n=62)$. $\mathrm{P}<0.0001$ by Kruskal-Wallis followed by Dunn's multiple comparisons post-test showed a significant difference only between the HD versus neutral, loss versus neutral and gain versus neutral group. For $A, B$, and $E$, $p$-values were obtained using a Mann-Whitney test and a Kruskal-Wallis one-way analysis of variance test for $F$ and data are presented as mean + SEM. P-values for $C$ and $D$ were obtained using a Student's $t$ tests and data are presented as mean + SD. ${ }^{*}, \mathrm{P}<0.05 ;{ }^{* *}, \mathrm{P}<0.005 ;{ }^{* * *}, \mathrm{P}<0.0005$.
RBPJ deficiency results in a Notch-like gene signature Because RBPJ acts as a transcriptional repressor, depletion can result in induction of target genes (Raafat et al., 2009; Liefke et al., 2010; Meier-Stiegen et al., 2010). We confirmed that RBPJ knockdown in MDA-MB-231 xenografts caused significant up-regulation of well characterized target genes of the HEY gene family (Fig. 3 A). HES1, SNAIL1, and GUCY1A3, additional known Notch target genes (Sahlgren et al., 2008; Borggrefe and Oswald, 2009; Chang et al., 2011), along with MMP1 were also up-regulated in RBPJ-depleted tumors (Fig. 3 B). To determine whether Notch activity contributes to transcriptional activation in the absence of RBPJ, we blocked activation of all Notch receptors in vitro using the $\gamma$-secretase inhibitor $N$-[N-(3,5-difluorophenacetyl)-Lalanyl]-S-phenylglycine t-butyl ester (DAPT) to prevent Notch receptor cleavage. We co-cultured MDA-MB-231 cells with Jagged1 ligand-expressing mouse cells to activate endogenous Notch. This induced HEY genes in control cells, and the induction was blocked by DAPT (Fig. 3 C). In contrast, up-regulation of HEY genes by RBPJ knockdown in ligand-stimulated cells was not affected by DAPT treatment, indicating up-regulation of these target genes independent of Notch receptor activation (Fig. 3 D). Similarly, HEY expression was significantly increased in DG75 $\mathrm{RBPJ}^{-/-}$tumors compared with RBPJexpressing controls and was not affected by DAPT-mediated Notch inhibition (unpublished data). These results imply that target gene expression is not solely an indicator of Notch receptor activity, as RBPJ depletion represents an alternate mechanism for promoting tumor growth and $H E Y$ expression.

To evaluate whether RBPJ loss produces a Notch-like gene signature, we identified genes differentially expressed in 10 human T-ALL cell lines with Notch activating mutations treated with vehicle or a Notch inhibitor (Benjamini-Hochberg corrected $\mathrm{P} \leq$ 0.05; Margolin et al., 2009). 170 genes showing a change in response to the Notch inhibitor across all T-ALL lines were defined as Notch-regulated genes (Table S2). Based on this signature, we assigned a Notch signature score (see Materials and methods) for each of the 828 invasive breast cancers from the TCGA dataset (shown in Fig. 1 C) comparing mean scores between samples with RBPJ loss to those without. Breast cancers with RBPJ loss had a significantly increased Notch signature score compared with tumors without RBPJ loss (Fig. 3 E). The breast cancer cases with RBPJ HD, copy loss, or copy gain had a significantly higher Notch signature score compared with samples with neutral RBPJ copy number (Fig. 3 F). These data imply that RBPJ activates a subset of Notch target genes.

\section{RBPJ depletion causes epigenetic changes that correspond to promoter activity}

To address how RBPJ loss leads to target gene activation, we first assessed whether another transcriptional activator binds 
A

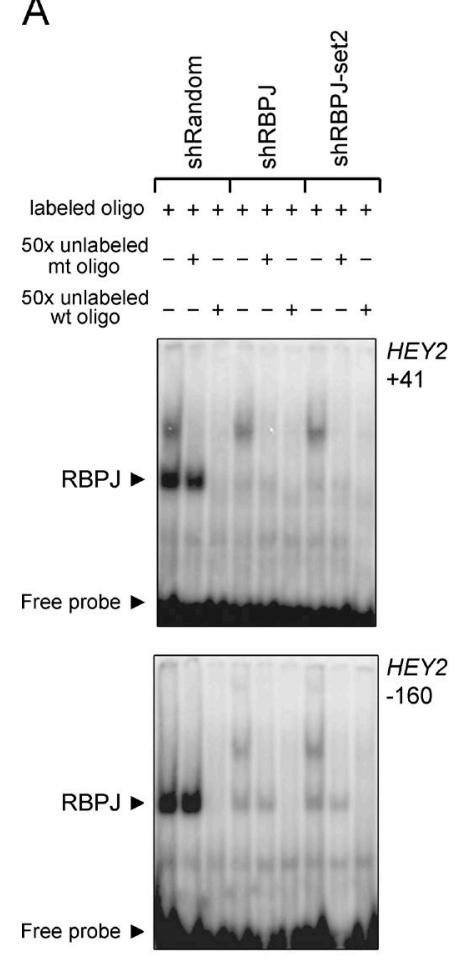

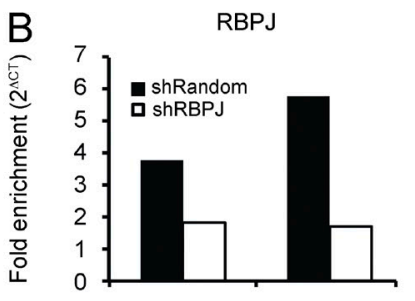
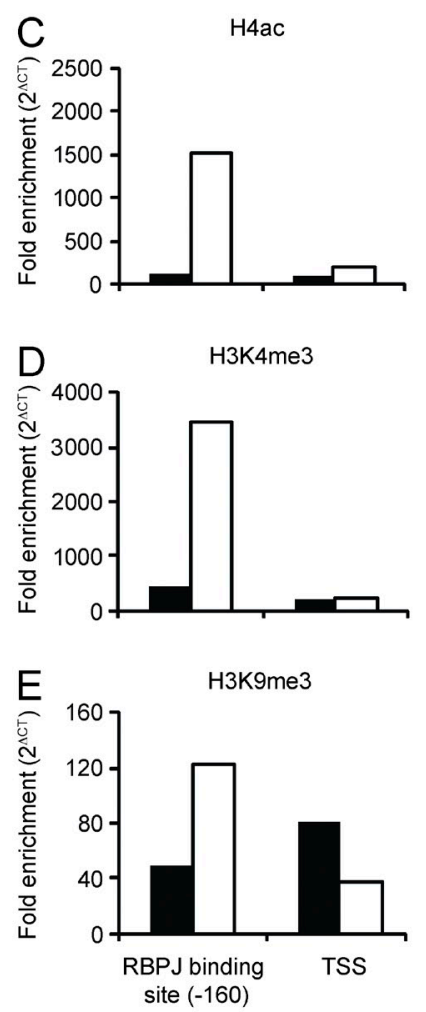
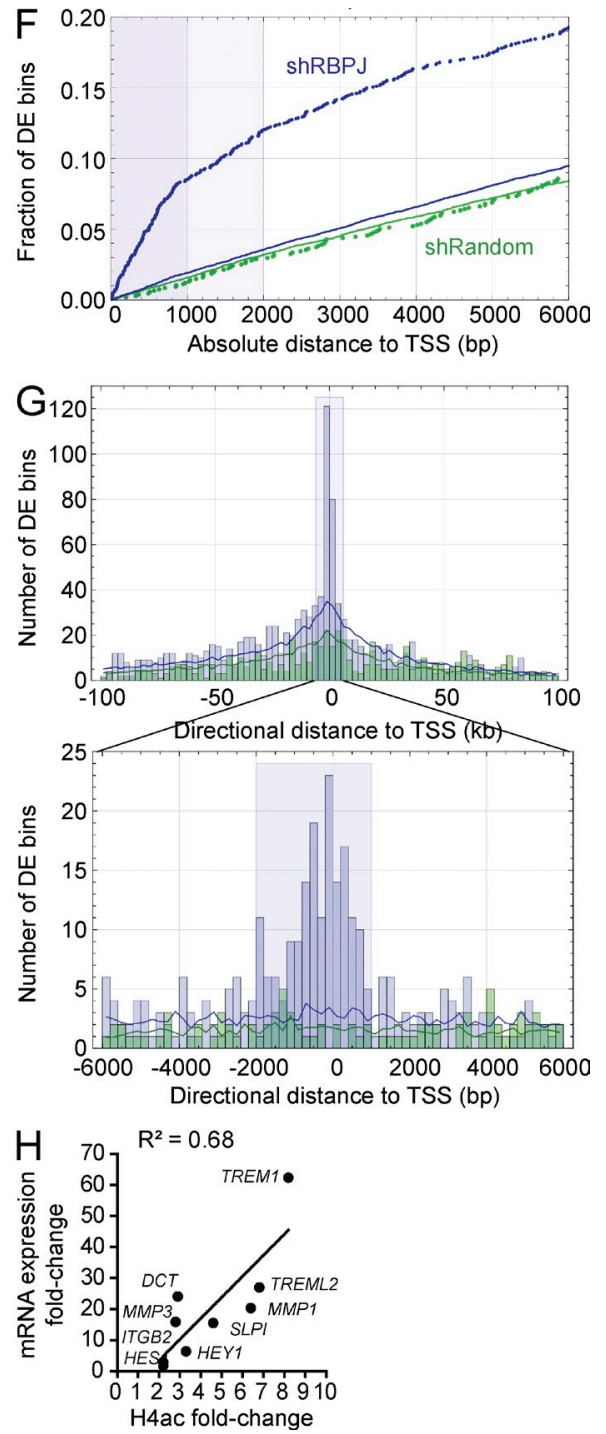

Figure 4. RBPJ depletion in MDA-MB-231 cells results in histone changes corresponding to promoter activity. (A) EMSA was performed using MDA-MB-231 nuclear lysates $(n=1)$ and labeled probes representing two RBPJ binding sites in the HEY2 promoter (HEY2: -160 to -167 and +41 to +48 bp from the TSS) in the absence or presence of excess unlabeled mutated probe (+mt) or excess wild-type probe (wt). Comparable results were obtained using binding sites in the HEY1 promoter (not depicted). (B-E) ChIP analysis of the HEY2 promoter $(n=1)$ with fold enrichment calculated as

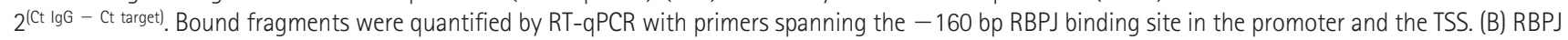
ChIP. (C) H4ac ChIP. (D) H3K4me3 ChIP. (E) H3K9me3 ChIP. (F and H) Locations of genome-wide 500 bp H4ac-differentially enriched regions identified by ChIP-seq (corrected $P \leq 0.005)$ relative to 38,405 nonredundant UCSC gene TSSs. Solid lines show random expectations $(n=20)$ for shRBPJ (blue) and shRandom (green). (F) Empirical distribution functions for the distance from the nearest TSS to the center of a differentially enriched (DE) region in the shRBPJ (blue) and the shRandom (green) control. Shaded blue regions that extend to 1 and $2 \mathrm{~kb}$ mark distances within which, for shRBPJ data, differentially enriched regions are spatially concentrated relative to TSSs. (G) Overview $( \pm 100 \mathrm{~kb})$ and detailed $( \pm 6 \mathrm{~kb})$ histograms of directional distance between a differentially enriched region center and its nearest TSS. The shaded blue rectangle in the detailed view marks the $-2 /+1 \mathrm{~kb}$ TSS regions used in analyses. $(\mathrm{H})$ Fold change in the H4ac signal correlates with fold change in mRNA expression (RT-qPCR) evaluated for a subset of the 114 TSSs that were significantly differentially enriched in the knockdown relative to shRandom cells, which correspond to 1 (Pearson correlation $\mathrm{P}=0.006$ ).

the vacant RBPJ motif in RBPJ-depleted cells. Electrophoretic mobility shift assays (EMSAs) in control and shRBPJ MDA-MB-231 cells did not reveal binding of alternate transcription factors to the RBPJ consensus sequences of the HEY1 (not depicted) and HEY2 promoters of RBPJ-depleted cells (Fig. 4 A). We also performed local chromatin immunoprecipitation (ChIP) in these cells confirming reduced RBPJ occupancy at the HEY2 promoter, corroborating the EMSA data (Fig. 4 B). Transcriptional repression by the RBPJ corepressor complex depends on histone deacetylase activity, which condenses chromatin and silences gene expression (Borggrefe and Oswald, 2009). To confirm HEY promoter activation, we demonstrated that RBPJ depletion resulted in histone acetylation by acetylated histone $\mathrm{H} 4$ (H4ac) ChIP followed by 

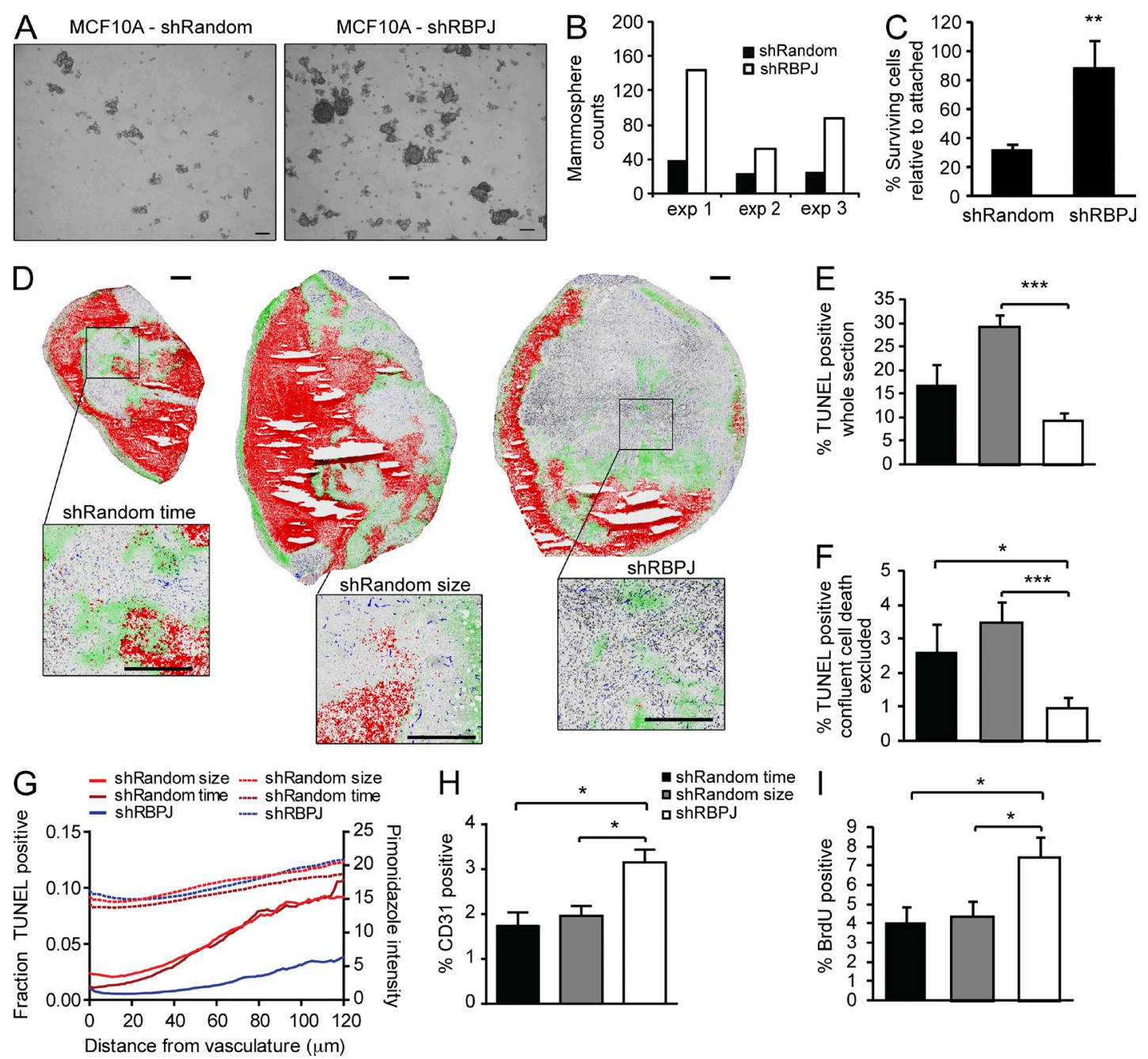

Figure 5. RBPJ deficiency increases mammosphere formation and confers resistance to cell death in breast cancer cells. (A and B) Mammosphere formation evaluated in MCF10A, a breast epithelial cell line. (A) Representative images of mammospheres formed on day 7 per 10,000 initiating cells from shRandom control cells and shRBPJ cells (bars, $100 \mu \mathrm{m}$ ). (B) Quantification of mammospheres from three independent experiments after $7 \mathrm{~d}$ of culture. (C) MDA-MB-231 cell survival in vitro after suspension for $48 \mathrm{~h}(n=4)$. (D) Staining and quantitative mapping of multiple markers in MDA-MB231 whole tumor cryosections. shRandom control tumors were harvested on the same day (time-matched), or when they reached comparable size (size-matched) to shRBPJ tumors. Representative images with TUNEL staining of dead cells (red), hypoxic areas with pimonidazole (green), S-phase proliferating cells with BrdU (black), vasculature with CD31 (blue) and gray tumor tissue boundaries with hematoxylin (bars, $500 \mu \mathrm{m}$ ). (E and F) Quantification of TUNEL staining shown in D ( $n=7$ for each group) in the whole section (E) or with confluent cell death excluded (F). (G) TUNEL labeling of cell death and pimonidazole staining of hypoxic cells was evaluated as a function of the distance from nearest CD31 stained vasculature. Lines represent averaged data for each group. Solid lines are TUNEL staining and dashed line are pimonidazole data ( $n=7$ for each group). (H and I) Quantification of CD31- $(H)$ and BrdU (I)-positive staining in MDA-MB-231 viable tumor tissue ( $n=7$ for each group). P-values for $C$ were obtained from a Student's $t$ test

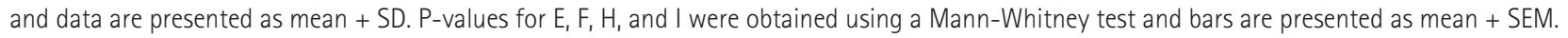
${ }^{*}, \mathrm{P} \leq 0.05 ;{ }^{* *}, \mathrm{P}<0.005 ;{ }^{* * *}, \mathrm{P} \leq 0.001$.

real-time quantitative PCR (RT-qPCR; Fig. 4 C). Histone $\mathrm{H} 3$ lysine 4 tri-methylation (H3K4me3), an alternate mark of active gene promoters, was similarly enriched at the RBPJ binding site of the HEY2 promoter in RBPJ-depleted cells (Fig. 4 D). Histone H3 lysine9 tri-methylation (H3K9me3), a modification associated with transcriptional repression, was reduced across the HEY2 transcription start site (TSS) in RBPJ-depleted cells (Fig. 4 E). The modest increase in H3K9me3 at the RBPJ binding site may result from feedback mechanisms after RBPJ depletion and HEY2 induction.
Hence, RBPJ loss effects histone marks at promoters of expressed genes.

To define derepressed/activated promoter regions in RBPJdepleted cells genome-wide, we profiled H4ac enrichment by ChIP-sequencing (seq), and then used edgeR (Robinson et al., 2010) to assess differential H4ac enrichment in 500 bp genome-wide regions in shRBPJ (knockdown) versus shRandom (control) MDA-MB-231 cells and retained regions that had a Benjamini-Hochberg corrected $\mathrm{P}<0.005$ (Benjamini and Hochberg, 1995). H4ac was differentially enriched in 
A
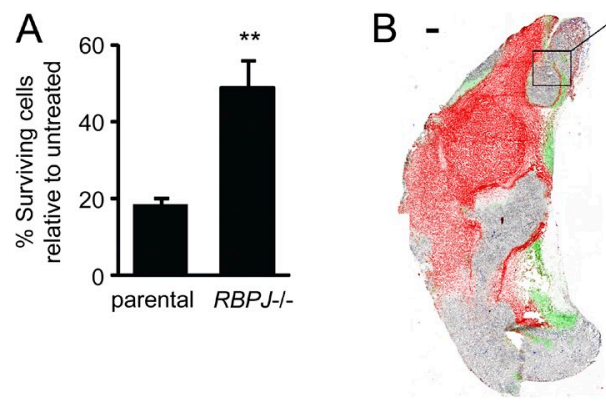

parental
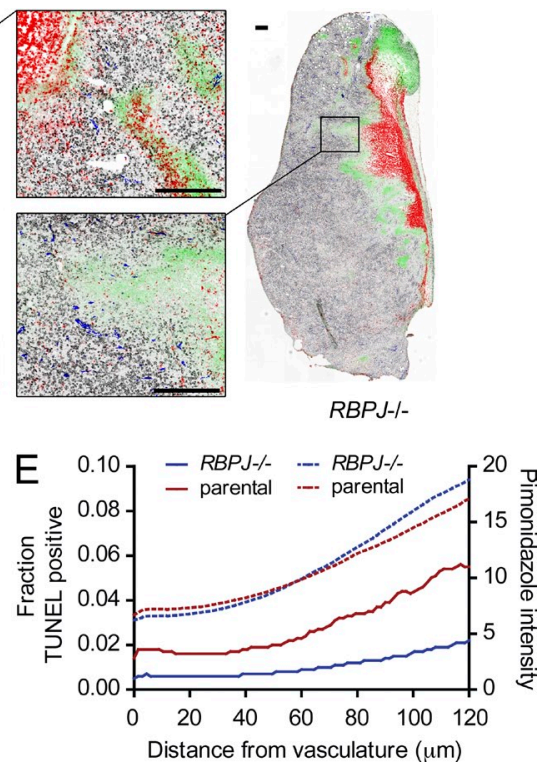

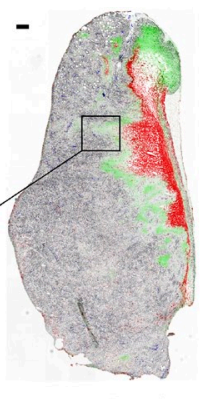

RBPJ-/-
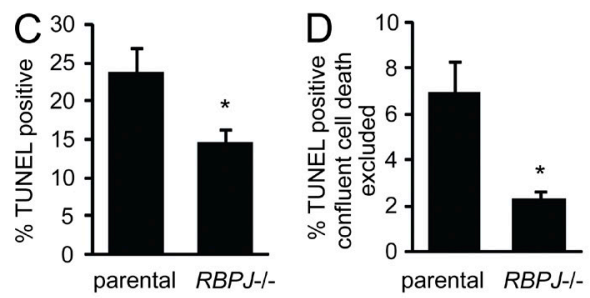

shRBPJ cells relative to shRandom in regions that extended from approximately $-2 \mathrm{~kb}$ to $+1 \mathrm{~kb}$ relative to TSSs, whereas for regions that were differentially enriched in the shRandom versus shRBPJ cells the spatial distribution was comparable to random expectation (Fig. 4, F and G). Consistent with this, in human $\mathrm{T}$ lymphoblastic leukemia cells the majority of RBPJ and Notch1 binding sites were found within $2 \mathrm{~kb}$ of TSSs (Wang et al., 2011). Assessing differential H4ac enrichment in $-2 /+1 \mathrm{~kb}$ regions for all 38,405 nonredundant UCSC gene TSSs, and retaining only regions that had an $\mathrm{H} 4 \mathrm{ac}$ fold change of at least 2 and a Benjamini-Hochberg corrected $\mathrm{P}<0.005$, we identified 114 TSS regions that were differentially enriched in RBPJ knockdown cells (Table S3). For nine of the differentially expressed genes, we used RT-qPCR to confirm that $\mathrm{H} 4 \mathrm{ac}$ enrichment was associated with increased mRNA expression (Fig. $4 \mathrm{H}$ ). Within regions $-2 /+1 \mathrm{~kb}$ from the TSS, eight of the nine genes had an RBPJ binding affinity score that was above the median for the global set of 38,405 regions, and for all nine TSS regions we identified a putative RBPJ binding site as a high-scoring match $(>0.96)$ to a TGGGAA de novo sequence motif (Aerts et al., 2005; Roider et al., 2007; unpublished data). To further evaluate whether promoters differentially marked with $\mathrm{H} 4 \mathrm{ac}$ are associated with genes previously identified as bound by RBPJ, we obtained 2,112 RBPJ binding sites in the CUTLL1 T-lymphoblastic leukemia/lymphoma cell line (Wang et al., 2011). Evaluating these RBPJ ChIP-seq peaks against our genome-wide $\mathrm{H} 4 \mathrm{ac}$ differentially enriched regions at an absolute distance of $2 \mathrm{~kb}$ from the TSS showed concordance in RBPJ knockdown regions $\left(\mathrm{P}=2.5 \times 10^{-4}\right)$ compared with control H4ac regions or random expectations ( $\mathrm{P}=0.11)$. Eight (HEY1, HES1,

UCKL1, PMEPA1, DGAT1, CR612603, MCF2L2, and ZDHHC14) of the 114 genes that we identified as differentially enriched in RBPJ knockdown cells at $-2 /+1 \mathrm{~kb}$ from the TSS have an RBPJ peak within $2 \mathrm{~kb}$ of the TSS. These results indicate that RBPJ depletion enriches for histone H4 acetylation near TSSs.

\section{RBPJ deficiency increases tumor cell survival}

To clarify how genes whose promoters were associated with $\mathrm{H} 4 \mathrm{ac}$ changes contribute to increased tumorigenicity, we used Ingenuity Pathway Analysis software to identify statistically significant biological functions for the genes corresponding to the 114 differentially enriched promoter regions in RBPJ knockdown cells. The 92 genes mapped by Ingenuity Pathway Analysis returned five significant cellular functions (BenjaminiHochberg corrected $\mathrm{P} \leq$ 0.025): lipid metabolism, molecular transport, small molecule biochemistry, cell death, and cellular compromise.

Because all of the induced genes that were marked with H4ac, except TREML2, appeared in the cell death function, we evaluated whether the apoptotic response was altered in RBPJ-depleted cells. We first performed mammosphere formation assays, which enrich for breast cancer stem cells with aberrant self-renewal, using a nontumorigenic breast epithelial cell line MCF10A transduced with shRandom and shRBPJ constructs. RBPJ knockdown increased mammosphere formation up to 3.7-fold (Fig. 5, A and B). Next, we incubated MDA-MB-231 cells in suspension for $48 \mathrm{~h}$ to induce anoikis. Compared with control cells, RBPJ knockdown markedly protected cells from death resulting from loss of cell matrix attachment (Fig. 5 C). To confirm that RBPJ 
deficiency also conferred a survival advantage in vivo, we used terminal deoxynucleotidyl transferase dUTP nick end labeling (TUNEL) to mark all dead cells in tumor cryosections. Both MDA-MB-231 size-matched shRandom control tumors, which were collected when they reached a comparable size to the RBPJ-knockdown tumors, and shRandom time-matched control tumors, which were harvested at the same time as the RBPJ-knockdown tumors, showed increased TUNEL staining compared with RBPJ-depleted tumors (Fig. 5, D-F).

We also stained for and quantified vasculature, hypoxic areas, and proliferating cells in tumor sections (Fig. 5, D and $\mathrm{G}-\mathrm{I})$. The difference in cell death between control and $R B P J$-depleted tumors was especially pronounced in regions at further distances from blood vessels where hypoxia is highest (Fig. 5 G). Microvessel density (quantified by CD31 staining) and proliferation (quantified by BrdU staining) was also significantly increased in shRBPJ tumors compared with both time-matched and size-matched control tumors (Fig. 5, $\mathrm{H}$ and $\mathrm{I}$ ).

We confirmed our findings in the DG75 lymphoma line, where $R B P J$ deficiency significantly reduced apoptosis in vitro and in vivo compared with parental cell xenografts (Fig. 6, A-E). This cell death was more pronounced at increasing distances from the vasculature, consistent with the findings in MDA-MB-231 xenografts (Fig. 6 E). However, no difference was observed in CD31 or BrdU staining in DG75 tumors (unpublished data). These data suggest that a common phenotype with RBPJ loss is an increase in survival of tumor cells in vivo.

\section{MYC and NF- $\mathrm{KB}$ contribute to increased survival in RBPJ-deficient cancer cells}

To identify transcription factors that may contribute to gene activation in the context of RBPJ depletion, we compared the spatial concordance between the genome-wide $500 \mathrm{bp} \mathrm{H} 4 \mathrm{ac}$ differentially enriched regions in MDA-MB-231 cells and the in vivo binding regions reported for 52 transcription factors in 24 cell lines (Fig. 7 A; Birney et al., 2007). Binding regions for MYC and NF-kB were concentrated within $\sim 1,000 \mathrm{bp}$ of the H4ac regions that were differentially enriched in the RBPJ knockdown cells (Materials and methods; Fig. 7 A). Evaluating 2,112 RBPJ binding sites from CUTLL T lymphoblastic leukemia/lymphoma cells (Wang et al., 2011), we also found enrichment of NF- $\mathrm{KB}$ and MYC binding regions (within $\sim 500$ bp of the binding site; Fig. 7, B and C). For the 114 genes that were differentially enriched in RBPJ knockdown cells, binding motifs for these two factors were among the most highly enriched within regions up to $1 \mathrm{~kb}$ upstream of the TSS (Zambelli et al., 2009; unpublished data). Both transcription factors have previously been shown to cooperatively regulate transcription of a common set of target genes with Notch (Lee et al., 2000; Margolin et al., 2009; Barbarulo et al., 2011).

To determine whether MYC and NF- $\mathrm{KB}-$ mediated activation are required to promote survival in $R B P J$-depleted cells, we performed anoikis assays in the presence of inhibitors against MYC (10058-F4) or NF-kB (BSM-345541, an I-Kappa-B kinase $\alpha$ inhibitor; Burke et al., 2003). Inhibition of either pathway abolished the survival advantage observed with RBPJ loss, resulting in cell death comparable to shRandom
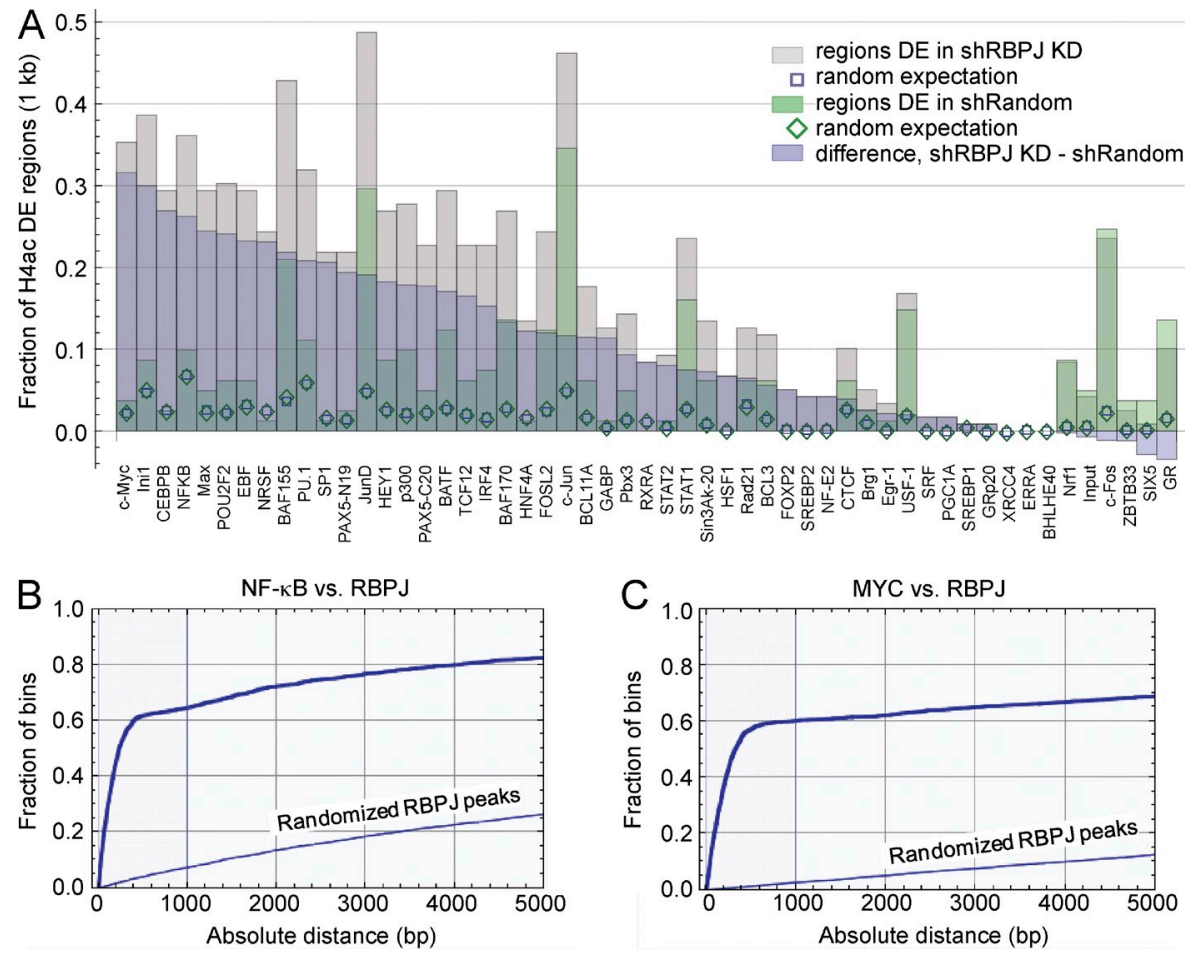

Figure 7. MYC and NF-кB binding regions are associated with $\mathrm{H} 4 \mathrm{ac}$ changes and are near RBPJ binding regions.

(A) Spatial association between genome-wide 500 bp H4ac regions in MDA-MB-231 cells and ENCODE ChIP-seq data for 52 transcription factors and input DNA in 24 cell lines (Birney et al., 2007). The bar chart shows the fractions of H4ac differentially enriched (DE) regions with centers within $1 \mathrm{~kb}$ of the center of an enriched transcription factor region. ( $B$ and $C$ ) Concordance between RBPJ ChIPSeq peaks and NF- $\mathrm{KB}$ and MYC binding regions. Spatial relationship between 2112 ChIP-seq enriched RBPJ peaks in CUTLL cells (Wang et al., 2011) and ChIP-seq enriched regions reported for NF- $\mathrm{B}$ p65 (B) and MYC (C) in ENCODE data (Birney et al., 2007). Empirical distribution functions for distances between the center of a thresholded ENCODE peak and the center of the nearest RBPJ peak region, contrasted to the distribution function when RBPJ peak locations on each chromosome were randomized. 
MDA-MB-231 cells (Fig. 8,A and B). BSM-345541 also significantly reduced $H E Y$ gene expression up-regulated by RBPJ knockdown (Fig. 8 C). To further define which NF-кB family members are required for survival activity in MDA-MB-231 cells, we used siRNA to deplete NF- $\mathrm{kB} 1$ (processed to p50), NF-кB2 (processed to p52), RELA/p65, RELB, and REL (c-Rel). Knockdown of either NFKB1 or the combined knockdown of RELB and REL abolished the survival advantage conferred by RBPJ depletion, suggesting that a combination of RELB/p50 and REL/p50 heterodimers may be responsible for the survival activity in shRBPJ MDA-MB-231 cells (Fig. 8 D). Finally, we observed a trend for enriched NF-кB (p50) and MYC binding at the HEY2 promoter in RBPJdepleted MDA-MB-231 cells (Fig. 8, E and F). Hence, loss of RBPJ-mediated repression may enable nuclear signaling by $\mathrm{NF}-\mathrm{\kappa B}$ and MYC activating prosurvival signaling pathways.

\section{DISCUSSION}

Our results indicate that depletion of RBPJ leads to gene induction of RBPJ-bound promoters, increased cell survival, and enhanced tumorigenicity. In contrast to NOTCH1, RBPJ expression is frequently reduced in several primary human tumor types including up to $33 \%$ of invasive breast cancer cases. We further show that RBPJ deficiency promotes tumor growth in two cancer cell lines derived from distinct tumor types in which RBPJ is targeted using two methods, shRNAmediated knockdown or genetic ablation. Although we present data on breast cancer and lymphoma as model systems, we provide additional clinical data supporting RBPJ loss in other human malignancies, such as lung cancers, through either or both RBPJ copy number loss or reduced mRNA expression. Supporting our observations, depletion of RBPJ was also reported to enhance eye tumor growth and metastases in Drosophila (Liefke et al., 2010).

RBPJ depletion causes a similar outcome to Notch activation in promoting tumor growth. Our current and previous work supports that retaining RBPJ transcriptional repression through blockade of Notch (by preventing Notch cleavage or transcriptional activation) inhibits MDA-MB-231 tumor growth (Leong et al., 2007). Conversely, RBPJ deficiency causes up-regulation of recognized Notch target genes, such as those of the HEY gene family, even in the presence of DAPT which inhibits Notch receptor processing and activation. We used a gamma secretase inhibitor to block signaling from all Notch receptors by preventing receptor activation at the membrane. Our aim was to perturb the Notch axis at a site upstream of the NotchIC-MAML-RBPJ ternary complex and show that the signal triggered by RBPJ loss is independent of
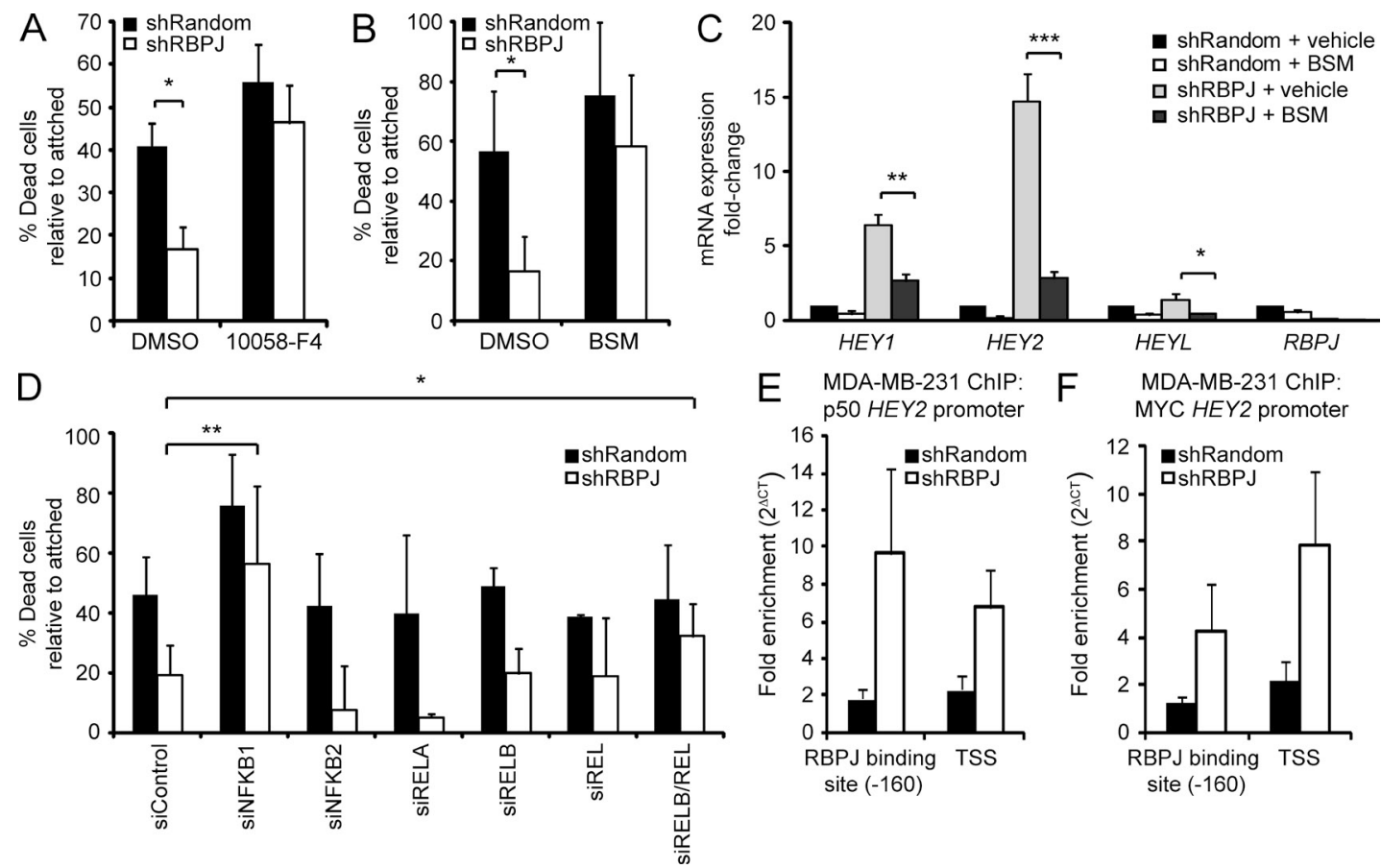

Figure 8. MYC and NF-кB contribute to survival in RBPJ-deficient MDA-MB-231 cells. (A and B) Cell death after forced suspension and treatment with indicated agents for $48 \mathrm{~h}$. (A) Vehicle control (DMSO) or $80 \mu \mathrm{M}$ 10058-F4 $(n=3)$. (B) Vehicle control (DMSO) or $25 \mu \mathrm{M}$ BSM-345541 (BSM, $n=4)$. (C) Gene expression analysis in MDA-MB-231 cells transduced with shRBPJ or shRandom after treatment with vehicle (DMSO) or $25 \mu \mathrm{M}$ BSM $(n=3)$. Data are expressed as fold-change relative to shRandom + vehicle. (D) Transfection with control small inhibitory (si)-RNA (siControl), siNFKB1, siNFKB2, siRELA, siRELB, and/or siREL $(n \geq 3)$. P-values are reported for treatments that significantly increased cell death in shRBPJ-expressing cells relative to the siControl. (E and F)

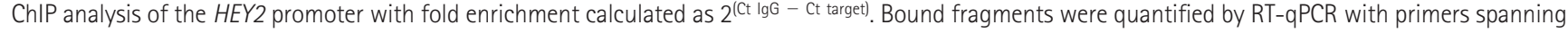
the -160 bp RBPJ binding site in the HEY2 promoter and the HEY2 TSS (E) NF-kB (p50) ChIP ( $n=5)$. (F) MYC ChIP $(n=3)$. P-values for A-D were obtained from a Student's $t$ test and data are presented as mean + SD. For $E$ and $F$, data are presented as SEM. ${ }^{*}, P<0.05 ;{ }^{* *}, P<0.005 ;{ }^{* * *}, P<0.0005$. 
Notch receptor activation. Derepression upon RBPJ removal also extends to other Notch receptor target genes. In our analysis of 828 invasive human breast cancers, cases with $R B P J$ loss were associated with an increased Notch signature score compared with tumors without RBPJ loss. Of these cases, breast cancers with RBPJ HD and copy loss had a significantly higher Notch signature score compared with samples with neutral RBPJ copy number. However, cases with RBPJ copy gain also showed an increased Notch signature score compared with samples with neutral RBPJ copy number, although RBPJ HD samples tended to have a higher Notch score than either of those with RBPJ copy loss or gain. In samples with gene amplification, increased RBPJ presumably facilitates transcriptional activation in response to Notch receptor activity. Together, our data support that target gene expression is not solely an indicator of Notch receptor activity, as RBPJ depletion represents an alternate mechanism for promoting tumor growth and $H E Y$ expression.

$\mathrm{RBPJ}$ is ubiquitously expressed in normal tissue having a promoter sequence characteristic of a housekeeping gene (Hamaguchi et al., 1992; Kawaichi et al., 1992). In the absence of Notch activation, RBPJ is a default transcriptional repressor and enters the nucleus precommitted to this function, which depends on histone deacetylase activity to condenses chromatin and silences gene expression (Zhou and Hayward, 2001; Borggrefe and Oswald, 2009). Conversely, our findings show that depletion of the RBPJ protein at RBPJ binding sites caused enrichment of both $\mathrm{H} 3 \mathrm{~K} 4 \mathrm{me} 3$ and $\mathrm{H} 4 \mathrm{ac}$, histone marks associated with active promoters. H3K9me3, a modification associated with transcriptional repression, was more modestly increased at the HEY2 RBPJ binding site but was reduced across the HEY2TSS in RBPJ knockdown cells. Our data support that $R B P J$ loss results in epigenetic changes that allow promoter activity. As preexisting RBPJ occupancy is required for gene repression in the absence of Notch activation at these promoters, these genes would be expected to be particularly susceptible to derepression upon RBPJ removal.

In Drosophila, active Notch can recruit RBPJ to promoters where it was previously not bound (Krejcí and Bray, 2007). RBPJ is likely not required for the repression of these genes but only for transcriptional activation via Notch. Thus loss of RBPJ may both derepress a subset of Notch target genes and potentially block Notch signaling at other targets. Further, Notch-independent RBPJ signaling has been reported. During early pancreatic and neural development, the Ptfla-RBPJ complex regulates gene transcription independently of Notch (Beres et al., 2006; Hori et al., 2008). In a human T lymphoblastic leukemia/lymphoma cell line, RBPJ sites were identified that do not bind Notch1. Only 36\% of Notch1 binding sites were found to overlap with RBPJ binding sites and 66\% of RBPJ sites overlapped with Notch1 sites as evaluated by ChIP-seq (Wang et al., 2011). Viral proteins can also co-opt RBPJ to allow for their own propagation via induction of viral and cellular promoters (Hayward, 2004; Persson and Wilson, 2010). Interestingly, fungal species lack Notch but have RBPJ genes (Prevorovský et al., 2009) providing additional support for a Notch-independent role for RBPJ. These Notch-independent genes may also be susceptible to derepression upon RBPJ depletion.

Several lines of evidence support that Notch is associated with conferring protection against cell death and is an important mediator of tumor cell survival (Capaccione and Pine, 2013). We have previously shown that expression of constitutively active Notch increases the resistance of MCF10A, a breast epithelial cell line, to anoikis and others have demonstrated induction of hyperproliferative acinar structures and colonies in soft agar (Leong et al., 2007; Mazzone et al., 2010). Compatible with a role in inhibiting apoptosis, mammary-specific deletion of Rbpj did not block Notch-induced tumor formation (Raafat et al., 2009). Rather, Notch-induced mammary tumors on a mammary-specific $R b p j^{-/-}$background showed reduced apoptosis compared with tumors containing functional Rbpj. Although the interpretation in this study was that Notch has Rbpj-independent oncogenic activity, our findings suggest that $R B P J$ loss prevents tumor cell death independent of Notch receptor activation, thereby promoting tumorigenesis. We observed reduced cell death as a common phenotype resulting from RBPJ deficiency in two distinct cancer models in vivo, which was especially pronounced in hypoxic areas of tumor tissue. These harsher tumor microenvironments have limited oxygen and nutrient supply and have been proposed to be the origin of more aggressive cells (Vaupel, 2004). RBPJ depletion may also contribute to increased proliferation and angiogenesis, as shRBPJ MDA-MB-231 tumors showed significantly increased BrdU and CD31 staining.

Loss of RBPJ-mediated repression may enable nuclear signaling by MYC and NF- $\mathrm{kB}$, which allows increased survival. Both these signaling pathways are thought to be activated in MDA-MB-231 cells and MYC and NF- $\mathrm{KB}$ have previously been shown to cooperatively regulate transcription of a common set of target genes with Notch (Nakshatri et al., 1997; Lee et al., 2000; Cappellen et al., 2007; Margolin et al., 2009; Barbarulo et al., 2011). In RBPJ-deficient cells, NF-кB and MYC binding was enriched at the HEY2 promoter and each of these pathways was required for protection against anoikis. Further, treatment with an inhibitor of NF- $\mathrm{kB}$ signaling reduced HEY gene expression up-regulated by RBPJ knockdown. Recently MYC has been shown to be a general amplifier of actively transcribed genes in tumor cells rather than binding and regulating a new set of genes (Lin et al., 2012). Hence MYC may amplify the output of the existing derepressed genes resulting from $R B P J$ knockdown. Our data suggest that in the absence of RBPJ, enhanced tumor cell survival is associated with MYC- and NF- $\mathrm{KB}$-driven prosurvival mechanisms.

Here, we describe a novel mechanism of cancer progression in mammalian cells that involves loss of RBPJ, the primary transcriptional mediator of canonical Notch signaling. The signal associated with loss of RBPJ overlaps with but is not identical to Notch activation. Indeed, Notch receptor activation is not required to drive the signal generated with $R B P J$ loss, but rather the signals are mediated in part by NF- $\mathrm{KB}$ and MYC. Although Notch activation and RBPJ depletion may 
not be equivalent, loss of $R B P J$ represents an alternate means of deregulating a component of the Notch signaling cascade, and so has therapeutic implications, as specific inhibition of Notch processing or function would be predicted to not have beneficial effects on tumors with RBPJ loss. Further, in cancers lacking RBPJ, targeting other oncogenic signals that contribute to survival, such as MYC or NF- $\mathrm{kB}$, may be a therapeutic option.

\section{MATERIALS AND METHODS}

Cell culture. The human DG75 Burkitt's lymphoma parental and $R B P J^{-/-}$ cell lines (Maier et al., 2005) were grown in RPMI 1640 medium (SigmaAldrich) supplemented with $10 \%$ heat-inactivated fetal bovine serum (HyClone), $2 \mathrm{mM}$ glutamine (Invitrogen), and $100 \mathrm{U}$ of each penicillin and streptomycin. MDA-MB-231 human breast carcinoma cells, viral producer line 293T, and AmphoPhoenix were cultured in DMEM (Sigma-Aldrich) and supplemented as above. For co-culture experiments, $10^{5} \mathrm{MDA}-\mathrm{MB}-231$ shRandom or shRBPJ cells were co-cultured with $10^{5}$ mouse SVEC4-10 endothelial cells transduced with MSCV-IRES-yellow fluorescent protein (MIY) control vector or MIY-Jagged1. Cells were plated in 12-well tissue culture dishes in media containing either $10 \mu \mathrm{M}$ DAPT (EMD Millipore) or DMSO vehicle control (Invitrogen). Cells were harvested after $48 \mathrm{~h}$ of coculture and RT-qPCR was performed with human-specific primers to avoid amplification of mouse transcripts. The normal breast epithelial cell line MCF10A was culture in a 1:1 mixture of DMEM/F12 supplemented with $5 \%$ horse serum, $2 \mathrm{mM}$ glutamine, $20 \mathrm{ng} / \mathrm{ml}$ epidermal growth factor (all from Sigma-Aldrich), $100 \mathrm{ng} / \mathrm{ml}$ cholera toxin (Cederlane), $10 \mu \mathrm{g} / \mathrm{ml}$ insulin (Sigma-Aldrich), $500 \mathrm{ng} / \mathrm{ml}$ hydrocortisone (Sigma-Aldrich), and $100 \mathrm{U} / \mathrm{ml}$ each of penicillin and streptomycin (Invitrogen). All cells were maintained at $37^{\circ} \mathrm{C}$ in a $5 \% \mathrm{CO}_{2}$ atmosphere.

Mammosphere culture. MCF10A human breast epithelial cells were suspended in MammoCult complete medium containing growth supplements heparin sulfate and hydrocortisone according to the manufacturer's instructions (Stem Cell Technologies). Mammospheres were cultured according to the MammoCult product sheet. In brief, the cell suspension was passed through a 45- $\mu \mathrm{m}$ cell strainer to obtain single cells. 10,000 MCF10A cells were seeded into each well of a 6-well ultra-low adherence dish (Stem Cell Technologies) containing $2 \mathrm{ml} \mathrm{MammoCult}$ complete medium. After $7 \mathrm{~d}$ in culture at $5 \% \mathrm{CO}_{2}$ at $37^{\circ} \mathrm{C}$, spheres $>60 \mu \mathrm{m}$ in diameter were counted.

Cell death assay. Anoikis experiments were performed with MDA-MB231 cells by plating $2.5 \times 10^{5}$ cells/well in Costar low-attachment 6-well plates (Stem Cell Technologies). Cells were then incubated in suspension for 48 h. BSM-345541 (EMD Biosciences) or 10058-F4 (Sigma-Aldrich) was added upon cell plating. In experiments where siRNA was used, cells were first transfected using Lipofectamine RNAiMax (Invitrogen) and then collected $24 \mathrm{~h}$ later for plating for the anoikis assay. AllStars negative control, NF-кB1 (Hs_NFkB1_7), NF-кB2 (Hs_NFkB2_9), RELA (Hs_RELA_7), RELB (Hs_RELB_5), and cREL (Hs_REL_5) siRNA were obtained from QIAGEN. For treatment with $100 \mu \mathrm{M}$ tert-butyl hydroperoxide (TBHP; Molecular Probes; Invitrogen), DG75 cells were plated at $7.5 \times 10^{5}$ cells/well in a 6-well plate and treated for $2 \mathrm{~h}$ to induce death. To quantify the surviving fraction, cells were harvested (for MDA-MB-231 cells the supernatant, PBS wash, and adherent cells were pooled). Cells were then pelleted, washed once with cold PBS, and resuspended in $100 \mu \mathrm{l}$ Annexin V binding buffer $(10 \mathrm{mM}$ Hepes, $140 \mathrm{mM} \mathrm{NaCl}$, and $2.5 \mathrm{mM} \mathrm{CaCl}_{2}, \mathrm{pH} 7.4$; all from Sigma-Aldrich). After addition of $5 \mu \mathrm{l}$ Annexin V-488 to DG75 cells or Annexin V-APC to MDA-MB-231 cells (both fluorescent conjugates from Invitrogen) and $5 \mu \mathrm{l}$ propidium iodide ( $1 \mathrm{mg} / \mathrm{ml}$; Sigma-Aldrich) per tube, the reaction was incubated for $15 \mathrm{~min} .500 \mu \mathrm{l}$ of ice-cold Annexin V binding buffer was added to each tube and flow cytometry analysis was performed immediately using the EPIC Elite flow cytometer (Beckman Coulter) and FCS Express (De Novo Software). Data are expressed as the proportion of surviving cells (defined as
Annexin V/propidium iodide negative) or dying cells (Annexin V and/or propidium iodide positive) relative to untreated attached (MDA-MB-231) or untreated (DG75) cells within each group.

RNA interference and gene transfer. shRNA-encoding DNA oligonucleotides (Integrated DNA Technologies) were cloned into the HpaI and Xhol site of the pLentilox3.7 green fluorescent protein (GFP) lentiviral vector (gift from L.Van Parijs, Massachusetts Institute of Technology, Cambridge, MA; Rubinson et al., 2003). The targeting sequences are as follows: shRandom, human shRBPJ, and shRBPJ-set2 have been previously described (Niessen et al., 2008; NM_015874); shScrambled, 5'-GATTAGAACCCTCACGGTACG-3' . For overexpression, the following retroviral vectors, all of which encoded human genes, were used: pLNCX (gift from A.D. Miller, Fred Hutchinson Cancer Research Centre, Seattle, WA), pLNC-FlagRBP2N, pLNC-FlagRBP2NR179H (the mutated RBPJ; MacKenzie et al., 2004), MIY, and pMIY-Jagged1. Target cells were transduced and purified based on GFP orYFP expression by flow sorting using a FACS-440 flow-sorter (BD). Cell lines expressing LNCX constructs were selected with $300 \mu \mathrm{g} / \mathrm{ml} \mathrm{G418}$ (Invitrogen). Knockdown and overexpression were assessed by RT-qPCR and immunoblotting.

RNA isolation and RT-qPCR. Total RNA was extracted using TRIzol (Invitrogen) according to the manufacturers' recommendations. First-strand cDNA was synthesized using Superscript II Reverse transcription (Invitrogen) and RT-qPCR was performed using the ABI Prism 7900HT detection system (Applied Biosystems) and the SYBR Green PCR Master Mix kit (Applied Biosystems), with gene-specific primer pairs (Table S4). Data obtained from the RT-qPCR reaction was analyzed using the comparative threshold cycle (Ct) method (User Bulletin No. 2; PerkinElmer Life Sciences) with GAPDH used as the reference gene for normalization.

Immunoblotting. Cultured cells and tumor tissues were lysed, resolved by SDS-PAGE, and probed overnight with primary antibodies using the following dilutions: 1:1,000 rat anti-RBPJ clone number 76719 (Institute of Immunology), and 1:10,000 mouse anti $\alpha$-tubulin (Sigma-Aldrich).

EMSA. Nuclear lysates were collected from shRandom, shRBPJ, and shRBPJset2 overexpressing MDA-MB-231 cells for the RBPJ EMSA assays. In brief, the cells were washed two times with PBS, resuspended in four pellet volumes of buffer A $(10 \mathrm{mM}$ Hepes-KOH, pH 7.8, $10 \mathrm{mM} \mathrm{KCl,} 1.5 \mathrm{mM}$ $\mathrm{MgCl}_{2}, 5 \mathrm{mM}$ with addition of a protease inhibitor cocktail; all from SigmaAldrich), pelleted for $15 \mathrm{~s}$ at $12,000 \mathrm{~g}$ and resuspended in $375 \mu \mathrm{l}$ buffer A containing $0.5 \% \mathrm{NP}-40$ (Sigma-Aldrich). After mixing, cells were incubated at $4^{\circ} \mathrm{C}$ for $10 \mathrm{~min}$ to lyse the cells, centrifuged for $10 \mathrm{~min}$ at $12,000 \mathrm{~g}$ at $4^{\circ} \mathrm{C}$, and the cytosolic extract was removed. The nuclei were washed once with buffer $\mathrm{A}$ and resuspended in three pellet volumes of buffer $\mathrm{B}(50 \mathrm{mM}$ Hepes$\mathrm{KOH}, \mathrm{pH} 7.8,10 \%$ glycerol, $50 \mathrm{mM} \mathrm{KCl}, 300 \mathrm{mM} \mathrm{NaCl}_{2}$, and $0.1 \mathrm{mM}$ EDTA, with addition of a protease inhibitor cocktail; all from SigmaAldrich) and incubated at $4^{\circ} \mathrm{C}$ for $20 \mathrm{~min}$. The nuclear lysates were collected after centrifugation for $10 \mathrm{~min}$ at $12,000 \mathrm{~g}$ at $4^{\circ} \mathrm{C}$ and stored at $-80^{\circ} \mathrm{C}$. The oligonucleotides containing RBPJ wild-type or mt motifs in the HEY2 promoter (Table S4) were end-labeled with ${ }^{32} \mathrm{P}$ dCTP using the Klenow fragment of DNA Polymerase I. The binding reaction $(10 \mathrm{mM}$ TrisHCl, $50 \mathrm{mM}$ $\mathrm{NaCl}, 1 \mathrm{mM}$ EDTA, pH 8, 4\% glycerol, and $2 \mu \mathrm{g}$ PolydI-dC binding buffer; all from Sigma-Aldrich), and $10 \mu \mathrm{g}$ of nuclear protein was performed by preincubating with either 50-fold excess wild-type or mt (Noseda et al., 2006) nonradioactive duplex oligos for $15 \mathrm{~min}$ on ice, and then adding a 150,000-cpm ${ }^{32} \mathrm{P}$-labeled double-stranded probe and incubating for $30 \mathrm{~min}$ at room temperature. DNA-protein complexes were electrophoresed on 5\% Tris-Borate EDTA gels, dried, and exposed to a phosphorimager plate for $16 \mathrm{~h}$.

Tumor xenografts. All protocols involving mice were evaluated and approved by the University of British Columbia Animal Care and Ethics Committee. Female non-obese diabetic/severe combined immunodeficient mice (NOD/SCID, Animal Resource Centre of the British Columbia Cancer 
Research Centre) were injected subcutaneously into the dorsa with human tumor cell lines. Tumor growth was monitored at the indicated times by external measurements with calipers, and tumor volume calculated using the formula $(\pi / 6 \times$ length $\times$ width $\times$ height $)$. For MDA-MB-231 tumor studies, $5 \times 10^{6}$ stably transduced cells were injected in $100 \mu$ l unsupplemented DMEM. For DG75 tumor studies, $10^{7}$ cells were injected in $200 \mu \mathrm{l} \mathrm{PBS}$. Individual tumors were split for either fixation in 4\% paraformaldehyde or freezing, and then used for histology, immunostaining, or RNA and protein analysis.

Whole section tumor staining. Tumor staining study protocols were similar to those previously reported (Kyle et al., 2003; Huxham et al., 2004). Mice were administered $1,500 \mathrm{mg} / \mathrm{kg} \mathrm{BrdU}$ (Sigma-Aldrich) in $0.9 \% \mathrm{NaCl}$ and $60 \mathrm{mg} / \mathrm{kg}$ pimonidazole (Hypoxyprobe) by intraperitoneal injection $2 \mathrm{~h}$ before tumor excision. Harvested tumors were frozen at $-20^{\circ} \mathrm{C}$ on an aluminum block, covered in embedding medium (O.C.T.) and stored at $-80^{\circ} \mathrm{C}$ until sectioning. Cryosections $10 \mu \mathrm{m}$ thick were obtained using a Cryosar HM560 (Microm International GmbH; Thermo Fisher Scientific), dried overnight, and fixed in 50:50 acetone-methanol for $10 \mathrm{~min}$ at room temperature. Incorporated $\mathrm{BrdU}$ was detected using a monoclonal mouse anti-BrdU antibody (clone BU33; Sigma-Aldrich), followed by an anti-mouse peroxidase-conjugated antibody (Sigma-Aldrich) and a metal-enhanced 3,3'-diaminobenzidine substrate (Pierce; Thermo Fisher Scientific). Hypoxic cells labeled with pimonidazole were detected using a polyclonal rabbit anti-pimonidazole antibody (Hypoxiprobe) and visualized with a fluorescent Alexa Fluor 488 tagged anti-rabbit secondary antibody.Vasculature was stained using a rat anti-mouse monoclonal antibody to CD31 (clone MEC 13.3; BD) and fluorescent Alexa Fluor 546 anti-rat secondary antibody (Invitrogen). TUNEL staining was performed using the in situ cell death detection kit (Roche) with a TMR red-tagged dUTP. After imaging of fluorescence, slides were placed in distilled water for $10 \mathrm{~min}$ and treated with $2 \mathrm{~mol} /$ liter $\mathrm{HCl}$ at room temperature for $1 \mathrm{~h}$, followed by neutralization for $5 \mathrm{~min}$ in $0.1 \mathrm{~mol} /$ liter sodium borate and rinsing in PBS. Slides were counterstained with hematoxylin, dehydrated, and mounted using Permount (Thermo Fisher Scientific) before being imaged. Images were acquired using a fluorescence microscope (Imager Z1; Carl Zeiss), a cooled, monochrome CCD camera (Retiga 4000R; QImaging), and motorized $x-y$ stage (Ludl Electronic Products). This system allows tiling of adjacent fields of view to compile images of entire tumor cryosections at a resolution of $0.75 \mu \mathrm{m} /$ pixel; sections were repeat-imaged at various points during staining. Using Image (National Institutes of Health) and user-supplied algorithms, images of BrdU, CD31, pimonidazole, TUNEL, and hematoxylin staining, all from the same tumor section, were overlaid and staining artifacts removed. Grayscale images of CD31 and TUNEL were inverted, thresholded, colorized (blue and red, respectively), and overlaid onto images of BrdU and hematoxylin (black and gray, respectively). Grayscale images of pimonidazole (green channel) were overlaid using Photoshop CS (version 8.0; Adobe) and combined using the multiply mask. Tumor tissues were analyzed either as whole tumor sections or as viable tissue only after crop and removal of confluent necrosis. TUNEL-positive staining was identified by selecting pixels a minimum of five standard deviations above tissue background levels and is reported as the fraction of TUNEL positive staining for whole sections or as the fraction of TUNEL-positive staining in viable tissue. BrdU-positive staining is reported as the fraction of positive pixels in viable tissue. Distribution analyses were performed in viable tissue only; each pixel in an image cropped to remove necrosis was sorted based on its distance relative to CD31-positive vasculature. For pimonidazole staining the mean pixel intensity is reported as a function of distance from vasculature, and for TUNEL analyses the fraction of pixels meeting or exceeding the stain threshold is reported as a function of distance from nearest vasculature.

Immunohistochemistry. The breast cancer tissue microarray sections were cut from formalin-fixed, paraffin-embedded small-core biopsies placed in the same block. Xenograft tumors excised from mice were fixed overnight in $10 \%$ phosphate-buffered formalin, progressively dehydrated through gradients of alcohol, and embedded in paraffin. Further analysis was performed by PMI Labs. In brief, 4- $\mu \mathrm{m}$ sections were cut, deparaffinized in xylene, rehydrated, and then stained with hematoxylin and eosin. For immunohistochemical analysis, antigen retrieval was performed with a Pascal decloaker (Dako) using citrate buffer, pH 6.0 (Dako). The rat anti-RBPJ7A11 antibody (Maier et al., 2005) and IgG2Bkappa control (BD) were used as primary antibodies at a 1:10 dilution. The slides were stained using the avidin-biotin-peroxidase method with the EnVision Flex kit (Dako) as per manufacturer's instructions, a 1:50 dilution of Biotin-SP-AffiniPure donkey anti-rat secondary (Medicorp Inc.), and 1:500 peroxidase-conjugated streptavidin (Medicorp Inc.) followed by the chromagen 3,3'-diaminobenzidine (Dako) to develop the immunostain. All sections were counterstained with hematoxylin. 1.5\% sodium bicarbonate was used as a bluing agent. Sections were imaged using a light microscope (BX41; Olympus). Tissue microarray slides were scanned with the imaging system (Aperio Technologies) and images were imported into ImageScope software (Aperio Technologies) for viewing and acquisition of micrographs. Immunophenotypic evaluation was performed independently by two evaluators who scored the tissue microarrays for presence or absence of RBPJ.

Microarray data. Published microarray data were obtained from the following sources: Gene Expression Omnibus (GEO) accession nos. GSE5364 (human breast cancer and adjacent nonmalignant tissue; Yu et al., 2008), GSE4824 (lung cancer cell lines; Lockwood et al., 2008), GSE3141 (111 NSCLC tumors; Bild et al., 2006); and the Oncomine database (normalized breast cancer microarray data; Ginestier et al., 2006). In addition, data for 67 normal human bronchial epithelial cells analyzed with the U133 plus array platform (Affymetrix), and 49 lung cancer samples analyzed with a custom chip (Affymetrix; Lockwood et al., 2010), were obtained from the System for Integrative Genomic Microarray Analysis (SIGMA) website (Chari et al., 2006). Microarray data were $\log 2$ transformed.

TCGA data. RNA-seq RPKM (z-score) and copy number (GISTIC alteration status) data for all genes of interest were downloaded for 828 invasive breast cancers, 178 lung SqCC, and 129 lung AC from the MSKCC cBIO Cancer Genomics Portal (Cerami et al., 2012; Gao et al., 2013). For each tumor type, the expression of RBPJ was compared between samples with RBPJ copy number loss versus those without using the Mann-Whitney U-Test (two-tailed).

Array comparative genomic hybridization (aCGH). DNA copy number profiles were generated for 49 lung cancer samples using whole genome tiling path array comparative genomic hybridization. Details of the genomic array, DNA extraction, labeling and hybridization, image analysis, and normalization have been described previously (Ishkanian et al., 2004). Data are available through the SIGMA website.

Notch signature score. We analyzed published microarray data (GEO accession no. GSE12868; Margolin et al., 2009) comparing 10 human T-ALL cell lines with Notch activating mutations treated with either DMSO or compound E. A total of 182 probes were identified as significantly downregulated by compound $\mathrm{E}$ treatment (Benjamini-Hochberg corrected $\mathrm{P} \leq$ 0.05 paired $t$ test, one-tailed; Table S2). Probes were mapped to 170 annotated genes, 162 of which had an RNA-seq RPKM z-score in TCGA. Expression data for each gene was compiled for 828 invasive breast cancers using a method modified from Loboda et al. (2010) to determine the Notch signature score. In brief, the mean expression for compound $\mathrm{E}$ down-regulated genes (i.e., Notch-induced genes) was determined for each sample. The mean expression of compound $\mathrm{E}$ up-regulated genes was then subtracted to give a composite Notch pathway score for each sample. The scores were then compared between breast cancer samples with RBPJ loss versus those without using the Mann-Whitney U-Test.

Histone, NF-кB, and MYC ChIP. shRandom or shRBPJ ChIP samples were prepared from stable MDA-MB-231 cell lines grown to $70 \%$ confluence. In brief, cells were cross-linked with $1 \%$ formaldehyde for $10 \mathrm{~min}$ at room temperature, the reaction was quenched by addition of $0.125 \mathrm{M}$ glycine, and 
the cells were then washed in PBS and harvested in the presence of protease inhibitors. ChIP was performed as previously described (Robertson et al., 2008). Chromatin DNA was fragmented by sonication for $10 \mathrm{~min}$ using the Sonic Dismembrator 550 (cup horn; Thermo Fisher Scientific) to produce chromatin fragments ranging from 100 to $800 \mathrm{bp}$. The chromatin was precleared with $40 \mu \mathrm{l}$ of blocked Protein A/G Sepharose beads (GE Healthcare) at $4^{\circ} \mathrm{C}$ for $2 \mathrm{~h}$. After centrifugation, supernatant was removed from the beads and transferred to fresh tubes. Each immunoprecipitation was performed with $100 \mu \mathrm{g}$ (histone IPs) or $25 \mu \mathrm{g}$ (transcription factor IPs) of precleared chromatin and $2.5 \mu \mathrm{g}$ of anti-H4ac antibody $(\mathrm{H} 4 \mathrm{~K} 5 / \mathrm{K} 8 / \mathrm{K} 12 / \mathrm{K} 16$, product number 06-866; EMD Millipore), $4 \mu \mathrm{l}$ rabbit anti-histone $\mathrm{H} 3$ trimethyl lysine 4 clone MC315 (Millipore), rabbit anti-histone H3 trimethyl lysine 9 (ab8898; Abcam), $2 \mu \mathrm{g}$ anti-NF-кB p50 antibody (H-119; Santa Cruz Biotechnology, Inc.), $2 \mu \mathrm{g}$ anti-c-MYC antibody (N-262; Santa Cruz Biotechnology, Inc.), or $4 \mu \mathrm{g}$ rabbit IgG from (EMD Millipore), and was incubated at $4^{\circ} \mathrm{C}$ for $1 \mathrm{~h}$. To each immunoprecipitation reaction, $20 \mu \mathrm{l}$ of protein $\mathrm{A} / \mathrm{G}$ Sepharose beads were added and incubated by rotating at $4^{\circ} \mathrm{C}$ overnight Beads were recovered by centrifugation and washed twice with ChIP wash buffer (20 mM Tris-HCl, pH 8.0, 0.1\% SDS, 1\% Triton X-100, 2 mM EDTA, and $150 \mathrm{mM} \mathrm{NaCl})$ and once with ChIP final wash buffer $(20 \mathrm{mM}$ Tris- $\mathrm{HCl}$, pH 8.0, 0.1\% SDS, $1 \%$ Triton X-100, $2 \mathrm{mM}$ EDTA, and $500 \mathrm{mM} \mathrm{NaCl}$ ). DNA-antibody complexes were eluted using $100 \mu$ Elution Buffer $(100 \mathrm{mM}$ $\mathrm{NaHCO}_{3}$ and $1 \% \mathrm{SDS}$ ), and $5 \mu \mathrm{g}$ DNase-free RNase (Roche) was added and incubated at $68^{\circ} \mathrm{C}$ for $2 \mathrm{~h}$ with shaking on a thermomixer to reverse the cross-links. The beads were pelleted by centrifugation and the supernatant was collected. Elution was repeated with the addition of $100 \mu \mathrm{l}$ of Elution Buffer and incubation at $68^{\circ} \mathrm{C}$ for 5 min with shaking on a thermomixer After pooling the two elutions, DNA was recovered from the eluate using the QIAquick PCR Purification kit (QIAGEN). The immunoprecipitated DNA was validated by qPCR using $0.25 \mu \mathrm{M}$ final concentration GAPDH primers (Forward Part\# 101221; Reverse Part \# 101222 from Active Motif), HEY2160, HEY2 TSS (Table S4), and ZNF3 as a negative control. qPCR was set up on a 7900HT Fast Real-Time PCR System (Applied Biosystems) using $1 \mu \mathrm{l}$ of immunoprecipitated eluate and the SYBR Green PCR Master Mix (Applied Biosystems). Cycling parameters were $95^{\circ} \mathrm{C}$ for $10 \mathrm{~min}, 40 \times\left(95^{\circ} \mathrm{C}\right.$ for $10 \mathrm{~s}$, $59^{\circ} \mathrm{C}$ for $30 \mathrm{~s}$, and $72^{\circ} \mathrm{C}$ for $\left.30 \mathrm{~s}\right), 72^{\circ} \mathrm{C}$ for $5 \mathrm{~min}, 95^{\circ} \mathrm{C}$ for $15 \mathrm{~s}, 59^{\circ} \mathrm{C}$ for $15 \mathrm{~s}$, and $95^{\circ} \mathrm{C}$ for $15 \mathrm{~s}$. Fold enrichment was calculated as $2^{\text {(Ct } \operatorname{IgG}-\mathrm{Ct} \text { target). }}$

RBPJ ChIP. shRandom or shRBPJ MDA-MB-231 cell lines were grown and processed for ChIP as described above with the following changes: chromatin preparation and immunoprecipitation steps were performed as described in the SimpleChIP Enzymatic Chromatin IP kit (Cell Signaling Technology) except using Protein A/G agarose beads (Promega). Each immunoprecipitation was performed with $20 \mu \mathrm{g}$ of precleared chromatin and $10 \mu \mathrm{l}$ rabbit anti-RBPSUH (Cell Signaling Technology), rabbit anti-histone $\mathrm{H} 3$ antibody, and rabbit anti-IgG (provided by kit). The immunoprecipitated DNA was validated by qPCR using primers for HEY2-160, HEY2 TSS (Table S4), and RPL30 as a negative control (provided by the kit). Fold en-

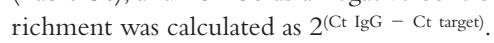

acH4 ChIP library construction and sequencing analysis. Sequencing libraries for the acH4 ChIP were prepared as previously described (Robertson et al., 2007). In brief, ChIP DNA (roughly $50 \mathrm{ng}$ ) was run in 8\% PAGE and the 100-300 bp fraction was excised, eluted overnight at $4^{\circ} \mathrm{C}$ in $300 \mu \mathrm{l}$ of elution buffer (5:1, LoTE buffer [3 mM Tris-HCl, pH 7.5, $0.2 \mathrm{mM}$ EDTA]$7.5 \mathrm{M}$ ammonium acetate), and purified by ethanol precipitation using a Spin-X Filter Tube (Thermo Fisher Scientific). Libraries were prepared using the Illumina single-end (SE) library construction protocol. This involved DNA end-repair, and phosphorylation by T4 DNA polymerase, Klenow DNA Polymerase, and T4 polynucleotide kinase, respectively, in a single reaction and subsequent $3^{\prime}$ A overhang generation by Klenow fragment $\left(3^{\prime}\right.$ to $5^{\prime}$ exo minus) and ligation to Illumina SE adapters (with $5^{\prime}$ overhangs). Adapterligated products were purified on Qiaquick spin columns (QIAGEN) and PCR-amplified using Phusion DNA polymerase for 10 cycles and the SE primer set (Illumina). The PCR product was purified using 8\% PAGE gels, and DNA quality was assessed and quantified using an Agilent DNA 1000 series II assay (Agilent Technologies) and a 7500 spectrophotometer (Nanodrop), and subsequently diluted to $10 \mathrm{nM}$. The final concentration was confirmed using a Quant-iT dsDNA HS assay kit and Qubit fluorometer (Invitrogen). Clusters were generated on Illumina cluster stations and were sequenced to $51 \mathrm{bp}$ for shRBPJ and $76 \mathrm{bp}$ for shRandom using GAIIx (Illumina), according to the manufacturer's instructions. The sequence read data were submitted to the Sequence Read Archive with accession no. SRP012092.

Identifying regions differentially enriched in H4ac. Single-end Illumina reads for shRBPJ (50 bp, $14.50 \mathrm{M}$ reads after MAPQ>10 filtering and duplicate removal) and shRandom (75 bp, $19.70 \mathrm{M}$ reads after MAPQ $>10$ filtering and duplicate removal) were aligned to the NCBI36/hg18 reference genome with BWA version 0.5.7 (Li et al., 2009). Differentially enriched 500-bp genome-wide regions were identified with edgeR (Robinson et al., 2010) v2.0.3 and R v2.11.1, using edgeR's TMM library size normalization to adjust for the different sequencing depths in the two libraries, and calculating $\mathrm{B}-\mathrm{H}$-corrected $\mathrm{p}$-values for differential enrichment. Considering both a concentration versus fold change plot that we annotated with adjusted $\mathrm{p}$-values and results from regions that we defined around transcriptional start sites (below), we set an adjusted p-value threshold of $0.005, \log _{2}$ fold change threshold of 1.0, and a $\log _{2}$ concentration threshold of -21.5 , and generated a set of filtered differentially enriched regions. The concentration threshold rejected regions with relatively low read coverages that would be most affected by variability in the ChIP-seq data.

Spatial relationship between differentially enriched regions and TSSs. For the above 500 bp differentially enriched regions, we determined distances between a region center and the nearest UCSC gene (Hsu et al., 2006) TSS. Spatial distributions were initially assessed as an empirical distribution function for the absolute value of distance from a differentially enriched region center to the nearest TSS (Fig. 4 F). The region in which H4ac was enriched was refined to $-2 /+1 \mathrm{~kb}$ by considering directional distances to a TSS, i.e., by considering transcript strand (Fig. 4 G). The calculations were done separately for regions that were differentially enriched in the shRBPJ versus the shRandom data, then for regions that were differentially enriched in the shRandom versus the shRBPJ data. Random expectations for both spatial distributions were generated as the mean of 20 distance calculations in which TSS locations on each chromosome were randomly relocated.

Identifying genes with TSS regions differentially enriched in H4ac. We used edgeR (Robinson et al., 2010) to identify which $-2 /+1 \mathrm{~kb}$ TSS regions were differentially enriched in the H4ac knockdown data. For this calculation, we addressed redundant TSS coordinates due to a gene having multiple transcripts by assigning sets of identical TSS coordinates to a single TSS coordinate, and merging any TSSs that were separated by $<500 \mathrm{bp}$ into a single coordinate ( $\mathrm{R}$ script available on request). Adjusted $\mathrm{p}$-values for published RPBJ target genes were assessed in the context of a scatter plot of regional read concentration versus fold change. From this, we set an adjusted p-value threshold of $0.005, a \log _{2}$ fold change threshold of 1.0 , and a $\log _{2}$ concentration threshold of -16 , and generated a set of 114 filtered, differentially expressed TSS regions. More stringent concentration filtering was chosen for TSS regions than for genome-wide bins because genome-wide bins were used to identify the extent of TSS regions, whereas thresholded TSS regions were used to define genes that were likely regulated by RBPJ binding.

Spatial relationship between differentially expressed regions and CHIPseq data. 55 ENCODE (Birney et al., 2007) ChIP-seq threshold region datasets were downloaded from the UCSC hg18 genome browser. Excluding Pol3 and TAF1 because they are not transcription factors (TFs), and using custom Mathematica scripts (Wolfram Research), we filtered each of the remaining $52 \mathrm{TF}$ datasets and 1 input DNA dataset by removing records for any region that had a score in the lowest 10\% for that TF or a length in the upper $10 \%$ for that TF. For each filtered dataset, we calculated distances between the center of a filtered, genome-wide $500 \mathrm{bp} \mathrm{H} 4 \mathrm{ac}$ differentially enriched region 
and the center of the nearest TF region. To identify spatial relationships that were shared or differed between TFs, we assessed empirical distribution functions. To compare results for H4ac regions that were differentially enriched in the knockdown or the control, and random expectations for each, we summarized the spatial relationships as a bar chart showing the Y-coordinate of each empirical distribution function at a center-to-center distance of $1 \mathrm{~kb}$ (Fig. $7 \mathrm{~A}$ ).

We used block bootstrap v0.8.1 (Encode Statistics) to estimate p-values for the concordance between locations of 2,112 genome-wide strong enriched regions from Wang et al. (2011) and locations of genome-wide 500-bp regions that edge $\mathrm{R}$ called as differentially enriched in $\mathrm{H} 4 \mathrm{ac}$ in the RBPJ knockdown (BH-corrected $\mathrm{P}<0.05)$ and in the control $(\mathrm{BH}$-corrected $\mathrm{P}<0.01$ ). For each pairwise comparison, we set $\mathrm{R}=0.05$ and reported the mean p-value from thirty 10,000 -iteration runs.

Transcription factor position frequency matrix (PFM) analysis. We generated a set of genome sequences for the 38,405 nonredundant $-2 /+1 \mathrm{~kb}$ TSS regions for NCBI36/hg18 UCSC genes that were used for the TSSregion $\mathrm{H} 4 \mathrm{ac}$ differential enrichment calculation (above). We used a commandline version of TRAP (Roider et al., 2007) to assign binding affinity scores to each TSS region, for each transcription factor binding matrix (PFM D'Haeseleer, 2006) in a set consisting of: all matrices in TRANSFAC Professional v9.3 (Wingender, 2008); a TGGGAA RBPJ motif and an NF-кB-like RBPJ motif, both returned by de novo discovery with MEME v3.5.7 (Bailey et al., 2009) from a set of 68 published, experimentally determined RBPJ binding site regions (Table S5); four NF- $\mathrm{kB}-$ related PFMs from the nonredundant subset of the JASPAR 2010 database (MA0107.1 RELA, MA0061.1 NF-кB, MA0105.1 NFKB1, and MA0101.1 REL; Portales-Casamar et al., 2010); and a published RelB-p52 PFM (Britanova et al., 2008). To identify high-scoring PFM matches, we scanned TSS region sequences with PFMs using MotifLocator v3.2, with default settings, and a third order background model that we generated from the 38405 nonredundant TSS regions using CreateBackgroundModel v3.2. We assessed enrichment of PFMs available in both JASPAR and the public TRANSFAC databases for the 114 knockdown differentially enriched genes, using the Pscan webserver (Zambelli et al., 2009) and $-1 / 0 \mathrm{~kb}$ TSS regions. Pscan ranks PFMs for enrichment of in a target set of TSS regions via the $\mathrm{P}$ values of $\mathrm{z}$-scores calculated for that target set versus a background whole-genome region set.

Ingenuity pathway analysis. IPA (Ingenuity Systems) was used to interpret acH4 data by performing Core Analysis using default parameters in the context of biofunctions (molecular and cellular functions). The 114 TSS regions that were identified as knockdown differentially enriched by the $\mathrm{H} 4 \mathrm{ac}$ analysis were defined as input parameters for the analysis. Significance was tested by with the $\mathrm{B}-\mathrm{H}$ correction for multiple testing using $\mathrm{P} \leq 0.025$.

Statistical analyses. Specific tests are noted in the text and figure legends. For pairwise comparisons, in vitro data were analyzed using a Student's $t$ test; otherwise, we used the Mann-Whitney $U$ test. The parametric one-way ANOVA and nonparametric Kruskal-Wallis one-way analysis of variance tests were used to compare multiple groups. Prism 6 (GraphPad Software) was used for statistical analysis and generating box-and-whisker plots. P-values $<0.05$ were considered significant unless otherwise stated.

Online supplemental material. Table $\mathrm{S} 1$ shows $R B P J$ copy number alteration in 216 cancer cell lines. Table S2 shows differentially expressed probes in duplicate cultures of 10 human T-ALL cell lines with Notch-activating mutations. Table S3 shows 114 transcriptional start sites differentially enriched in H4ac in RBPJ knockdown MDA-MB-231 cells. Table S4 shows a list of primer sequences used. Table S5 shows RBPJ binding regions used for PFM analysis Online supplemental material is available at http://www.jem .org/cgi/content/full/jem.20121192/DC1.

We thank Fred Wong and Denise McDougal for assistance with flow cytometry and cell sorting and Patrick Plettner for assistance with data submission.
This research was supported by grants to A. Karsan from the Canadian Institutes of Health Research (MOP 64354) and the Cancer Research Society. I. Kulic, L. Chang, and J.H.E. Baker were supported by Studentships from the Michael Smith Foundation for Health Research. I. Kulic was also supported by a Doctoral Research Award from the Canadian Institutes of Health Research and a Postgraduate Master's Scholarship from the Natural Sciences and Engineering Research Council of Canada. B. Kempkes was supported by a grant from the Deutsche Jose Carreras Leukämie-Stiftung (R02/11) and the Deutsche Krebshilfe (106899).

The authors declare no competing financial interests.

Contributions: I. Kulic designed and performed experiments, analyzed and interpreted data, and wrote the manuscript. G. Robertson wrote the manuscript and conducted bioinformatic analysis of ChIP-seq data with assistance from I. Kulic, L. Chang, and M.D. Robinson using the tool developed by M.D. Robinson. H-J. Chun and S. Jones also contributed to bioinformatic analysis. W.W. Lockwood conducted analysis on the Notch signature, TCGA, and aCGH data. L. Chang, J.H.E. Baker, W. Mok, M. Fuller, M. Fournier, and V. Chou performed experiments. A.I. Minchinton enabled whole section tumor staining. Cell lines were provided by B. Kempkes and W.L. Lam and tissue microarrays by B. Gilks and T.A. Thomson. M. Hirst and M. Marra enabled generation of ChIP-seq data. A. Karsan conceived and supervised the project, interpreted data, and wrote the manuscript.

Submitted: 2 June 2012

Accepted: 20 November 2014

\section{REFERENCES}

Aerts, S., P.Van Loo, G. Thijs, H. Mayer, R. de Martin, Y. Moreau, and B. De Moor. 2005. TOUCAN 2: the all-inclusive open source workbench for regulatory sequence analysis. Nucleic Acids Res. 33:W393-W396. http:// dx.doi.org/10.1093/nar/gki354

Bailey, T.L., M. Boden, F.A. Buske, M. Frith, C.E. Grant, L. Clementi, J. Ren, W.W. Li, and W.S. Noble. 2009. MEME SUITE: tools for motif discovery and searching. Nucleic Acids Res. 37:W202-W208. http://dx.doi .org/10.1093/nar/gkp335

Barbarulo, A., P. Grazioli, A.F. Campese, D. Bellavia, G. Di Mario, M. Pelullo, A. Ciuffetta, S. Colantoni, A.Vacca, L. Frati, et al. 2011. Notch3 and canonical NF- $\mathrm{kB}$ signaling pathways cooperatively regulate Foxp3 transcription. J. Immunol. 186:6199-6206. http://dx.doi.org/10.4049/jimmunol.1002136

Benjamini, Y., and Y. Hochberg. 1995. Controlling the False Discovery Rate: A Practical and Powerful Approach to Multiple Testing. Journal of the Royal Statistical Society, Series B. 57:289-300.

Beres, T.M., T. Masui, G.H. Swift, L. Shi, R.M. Henke, and R.J. MacDonald. 2006. PTF1 is an organ-specific and Notch-independent basic helixloop-helix complex containing the mammalian Suppressor of Hairless (RBP-J) or its paralogue, RBP-L. Mol. Cell. Biol. 26:117-130. http:// dx.doi.org/10.1128/MCB.26.1.117-130.2006

Bild, A.H., G. Yao, J.T. Chang, Q. Wang, A. Potti, D. Chasse, M.B. Joshi, D. Harpole, J.M. Lancaster, A. Berchuck, et al. 2006. Oncogenic pathway signatures in human cancers as a guide to targeted therapies. Nature. 439:353-357. http://dx.doi.org/10.1038/nature04296

Birney, E., J.A. Stamatoyannopoulos, A. Dutta, R. Guigó, T.R. Gingeras, E.H. Margulies, Z. Weng, M. Snyder, E.T. Dermitzakis, R.E. Thurman, et al. Children's Hospital Oakland Research Institute. 2007. Identification and analysis of functional elements in 1\% of the human genome by the ENCODE pilot project. Nature. 447:799-816. http://dx.doi.org/10 $.1038 /$ nature 05874

Borggrefe, T., and F. Oswald. 2009. The Notch signaling pathway: transcriptional regulation at Notch target genes. Cell. Mol. Life Sci. 66:1631-1646. http://dx.doi.org/10.1007/s00018-009-8668-7

Bray, S.J. 2006. Notch signalling: a simple pathway becomes complex. Nat. Rev. Mol. Cell Biol. 7:678-689. http://dx.doi.org/10.1038/nrm2009

Britanova, L.V., V.J. Makeev, and D.V. Kuprash. 2008. In vitro selection of optimal RelB/p52 DNA-binding motifs. Biochem. Biophys. Res. Commun. 365:583-588. http://dx.doi.org/10.1016/j.bbrc.2007.10.200

Burke, J.R., M.A. Pattoli, K.R. Gregor, P.J. Brassil, J.F. MacMaster, K.W. McIntyre, X. Yang, V.S. Iotzova, W. Clarke, J. Strnad, et al. 2003. BMS345541 is a highly selective inhibitor of IкB kinase that binds at an allosteric site of the enzyme and blocks NF-кB-dependent transcription 
in mice. J. Biol. Chem. 278:1450-1456. http://dx.doi.org/10.1074/jbc M209677200

Capaccione, K.M., and S.R. Pine. 2013. The Notch signaling pathway as a mediator of tumor survival. Carcinogenesis. 34:1420-1430. http://dx.doi .org/10.1093/carcin/bgt127

Cappellen, D., T. Schlange, M. Bauer, F. Maurer, and N.E. Hynes. 2007. Novel c-MYC target genes mediate differential effects on cell proliferation and migration. EMBO Rep. 8:70-76. http://dx.doi.org/10.1038/ sj.embor.7400849

Cerami, E., J. Gao, U. Dogrusoz, B.E. Gross, S.O. Sumer, B.A. Aksoy, A. Jacobsen, C.J. Byrne, M.L. Heuer, E. Larsson, et al. 2012. The cBio cancer genomics portal: an open platform for exploring multidimensional cancer genomics data. Cancer Discov. 2:401-404. http://dx.doi.org/10 .1158/2159-8290.CD-12-0095

Chang, A.C., Y. Fu, V.C. Garside, K. Niessen, L. Chang, M. Fuller, A. Setiadi, J. Smrz, A. Kyle, A. Minchinton, et al. 2011. Notch initiates the endothelialto-mesenchymal transition in the atrioventricular canal through autocrine activation of soluble guanylyl cyclase. Dev. Cell. 21:288-300. http://dx .doi.org/10.1016/j.devcel.2011.06.022

Chari, R., W.W. Lockwood, B.P. Coe, A. Chu, D. Macey, A. Thomson, J.J. Davies, C. MacAulay, and W.L. Lam. 2006. SIGMA: a system for integrative genomic microarray analysis of cancer genomes. BMC Genomics. 7:324. http://dx.doi.org/10.1186/1471-2164-7-324

D'haeseleer, P. 2006. What are DNA sequence motifs? Nat. Biotechnol. 24 423-425. http://dx.doi.org/10.1038/nbt0406-423

Gao, J., B.A. Aksoy, U. Dogrusoz, G. Dresdner, B. Gross, S.O. Sumer, Y. Sun, A. Jacobsen, R. Sinha, E. Larsson, et al. 2013. Integrative analysis of complex cancer genomics and clinical profiles using the cBioPortal. Sci. Signal. 6:p11. http://dx.doi.org/10.1126/scisignal.2004088

Ginestier, C., N. Cervera, P. Finetti, S. Esteyries, B. Esterni, J. Adélaïde, L. Xerri, P.Viens, J. Jacquemier, E. Charafe-Jauffret, et al. 2006. Prognosis and gene expression profiling of 20q13-amplified breast cancers. Clin. Cancer Res. 12:4533-4544. http://dx.doi.org/10.1158/1078-0432.CCR-05-2339

Hamaguchi, Y., Y. Yamamoto, H. Iwanari, S. Maruyama, T. Furukawa, N. Matsunami, and T. Honjo. 1992. Biochemical and immunological characterization of the DNA binding protein (RBP-Jк) to mouse J kappa recombination signal sequence. J. Biochem. 112:314-320.

Hayward, S.D. 2004.Viral interactions with the Notch pathway. Semin. Cancer Biol. 14:387-396. http://dx.doi.org/10.1016/j.semcancer.2004.04.018

Hori, K., J. Cholewa-Waclaw, Y. Nakada, S.M. Glasgow, T. Masui, R.M. Henke, H. Wildner, B. Martarelli, T.M. Beres, J.A. Epstein, et al. 2008. A nonclassical bHLH Rbpj transcription factor complex is required for specification of GABAergic neurons independent of Notch signaling. Genes Dev. 22:166-178. http://dx.doi.org/10.1101/gad.1628008

Hsu, F., W.J. Kent, H. Clawson, R.M. Kuhn, M. Diekhans, and D. Haussler. 2006. The UCSC Known Genes. Bioinformatics. 22:1036-1046. http:// dx.doi.org/10.1093/bioinformatics/bt1048

Huxham, L.A., A.H. Kyle, J.H. Baker, L.K. Nykilchuk, and A.I. Minchinton. 2004. Microregional effects of gemcitabine in HCT-116 xenografts. Cancer Res. 64:6537-6541. http://dx.doi.org/10.1158/0008-5472.CAN-04-0986

Ishkanian, A.S., C.A. Malloff, S.K. Watson, R.J. DeLeeuw, B. Chi, B.P. Coe, A. Snijders, D.G.Albertson, D. Pinkel, M.A. Marra, et al. 2004. A tiling resolution DNA microarray with complete coverage of the human genome. Nat. Genet. 36:299-303. http://dx.doi.org/10.1038/ng1307

Kawaichi, M., C. Oka, S. Shibayama, A.E. Koromilas, N. Matsunami, Y. Hamaguchi, and T. Honjo. 1992. Genomic organization of mouse JK recombination signal binding protein (RBP-Jк) gene. J. Biol. Chem. 267: 4016-4022.

Koch, U., and F. Radtke. 2007. Notch and cancer: a double-edged sword. Cell. Mol. Life Sci. 64:2746-2762. http://dx.doi.org/10.1007/s00018-007-7164-1

Kovall, R.A., and W.A. Hendrickson. 2004. Crystal structure of the nuclear effector of Notch signaling, CSL, bound to DNA. EMBO J. 23:34413451. http://dx.doi.org/10.1038/sj.emboj.7600349

Krejcí, A., and S. Bray. 2007. Notch activation stimulates transient and selective binding of $\mathrm{Su}(\mathrm{H}) / \mathrm{CSL}$ to target enhancers. Genes Dev. 21:13221327. http://dx.doi.org/10.1101/gad.424607

Kyle, A.H., L.A. Huxham, J.H. Baker, H.E. Burston, and A.I. Minchinton. 2003. Tumor distribution of bromodeoxyuridine-labeled cells is strongly dose dependent. Cancer Res. 63:5707-5711.
Lee, S.H., X. Wang, and J. DeJong. 2000. Functional interactions between an atypical NF- $\mathrm{\kappa B}$ site from the rat CYP2B1 promoter and the transcriptional repressor RBP-Jк/CBF1. Nucleic Acids Res. 28:2091-2098. http://dx.doi.org/10.1093/nar/28.10.2091

Leong, K.G., K. Niessen, I. Kulic, A. Raouf, C. Eaves, I. Pollet, and A. Karsan. 2007. Jagged1-mediated Notch activation induces epithelial-to-mesenchymal transition through Slug-induced repression of E-cadherin. J. Exp. Med. 204:2935-2948. http://dx.doi.org/10.1084/jem.20071082

Li, J., F. Gao, N. Li, S. Li, G.Yin, G. Tian, S. Jia, K. Wang, X. Zhang, H.Yang, et al. 2009. An improved method for genome wide DNA methylation profiling correlated to transcription and genomic instability in two breast cancer cell lines. BMC Genomics. 10:223. http://dx.doi.org/10.1186/ 1471-2164-10-223

Liefke, R., F. Oswald, C. Alvarado, D. Ferres-Marco, G. Mittler, P. Rodriguez, M. Dominguez, and T. Borggrefe. 2010. Histone demethylase KDM5A is an integral part of the core Notch-RBP-J repressor complex. Genes Dev. 24:590-601. http://dx.doi.org/10.1101/gad.563210

Lin, C.Y., J. Lovén, P.B. Rahl, R.M. Paranal, C.B. Burge, J.E. Bradner, T.I. Lee, and R.A. Young. 2012. Transcriptional amplification in tumor cells with elevated c-Myc. Cell. 151:56-67. http://dx.doi.org/10.1016/j.cell.2012.08.026

Loboda, A., M. Nebozhyn, R. Klinghoffer, J. Frazier, M. Chastain, W. Arthur, B. Roberts, T. Zhang, M. Chenard, B. Haines, et al. 2010. A gene expression signature of RAS pathway dependence predicts response to PI3K and RAS pathway inhibitors and expands the population of RAS pathway activated tumors. BMC Med. Genomics. 3:26. http://dx.doi.org/ 10.1186/1755-8794-3-26

Lockwood, W.W., R. Chari, B.P. Coe, L. Girard, C. Macaulay, S. Lam, A.F. Gazdar, J.D. Minna, and W.L. Lam. 2008. DNA amplification is a ubiquitous mechanism of oncogene activation in lung and other cancers. Oncogene. 27:4615-4624. http://dx.doi.org/10.1038/onc.2008.98

Lockwood, W.W., R. Chari, B.P. Coe, K.L. Thu, C. Garnis, C.A. Malloff, J. Campbell, A.C. Williams, D. Hwang, C.Q. Zhu, et al. 2010. Integrative genomic analyses identify BRF2 as a novel lineage-specific oncogene in lung squamous cell carcinoma. PLoS Med. 7:e1000315.http://dx.doi.org/ 10.1371/journal.pmed.1000315

Lockwood, W.W., I.M. Wilson, B.P. Coe, R. Chari, L.A. Pikor, K.L. Thu, L.M. Solis, M.I. Nunez, C. Behrens, J.Yee, et al. 2012. Divergent genomic and epigenomic landscapes of lung cancer subtypes underscore the selection of different oncogenic pathways during tumor development. PLoS ONE. 7:e37775. http://dx.doi.org/10.1371/journal.pone.0037775

MacKenzie, F., P. Duriez, B. Larrivée, L. Chang, I. Pollet, F.Wong, C.Yip, and A. Karsan. 2004. Notch4-induced inhibition of endothelial sprouting requires the ankyrin repeats and involves signaling through RBP-Jк. Blood. 104:1760-1768. http://dx.doi.org/10.1182/blood-2003-12-4244

Maier, S., M. Santak, A. Mantik, K. Grabusic, E. Kremmer, W. Hammerschmidt, and B. Kempkes. 2005. A somatic knockout of CBF1 in a human B-cell line reveals that induction of CD21 and CCR7 by EBNA-2 is strictly CBF1 dependent and that downregulation of immunoglobulin $\mathrm{M}$ is partially CBF1 independent. J. Virol. 79:8784-8792. http://dx.doi.org/10 $.1128 /$ JVI.79.14.8784-8792.2005

Margolin, A.A., T. Palomero, P. Sumazin, A. Califano, A.A. Ferrando, and G. Stolovitzky. 2009. ChIP-on-chip significance analysis reveals large-scale binding and regulation by human transcription factor oncogenes. Proc. Natl. Acad. Sci. USA. 106:244-249. http://dx.doi.org/10.1073/pnas .0806445106

Mazzone, M., L.M. Selfors, J. Albeck, M. Overholtzer, S. Sale, D.L. Carroll, D. Pandya, Y. Lu, G.B. Mills, J.C. Aster, et al. 2010. Dose-dependent induction of distinct phenotypic responses to Notch pathway activation in mammary epithelial cells. Proc. Natl. Acad. Sci. USA. 107:5012-5017. http://dx.doi.org/10.1073/pnas.1000896107

Meier-Stiegen, F., R. Schwanbeck, K. Bernoth, S. Martini, T. Hieronymus, D. Ruau, M. Zenke, and U. Just. 2010. Activated Notch1 target genes during embryonic cell differentiation depend on the cellular context and include lineage determinants and inhibitors. PLoS ONE. 5:e11481. http://dx.doi.org/10.1371/journal.pone.0011481

Nakshatri, H., P. Bhat-Nakshatri, D.A. Martin, R.J. Goulet Jr., and G.W. Sledge Jr. 1997. Constitutive activation of NF-kB during progression of breast cancer to hormone-independent growth. Mol. Cell. Biol. 17:3629-3639. 
Network, T.C.G.A. Cancer Genome Atlas Network. 2012. Comprehensive molecular portraits of human breast tumours. Nature. 490:61-70. http:// dx.doi.org/10.1038/nature11412

Niessen, K.,Y. Fu, L. Chang, P.A. Hoodless, D. McFadden, and A. Karsan. 2008 Slug is a direct Notch target required for initiation of cardiac cushion cellularization. J. Cell Biol. 182:315-325. http://dx.doi.org/10.1083/ jcb. 200710067

Noseda, M.,Y. Fu, K. Niessen, F. Wong, L. Chang, G. McLean, and A. Karsan. 2006. Smooth Muscle $\alpha$-actin is a direct target of Notch/CSL. Circ Res. 98:1468-1470. http://dx.doi.org/10.1161/01.RES.0000229683 .81357 .26

Persson, L.M., and A.C. Wilson. 2010. Wide-scale use of Notch signaling factor CSL/RBP-JK in RTA-mediated activation of Kaposi's sarcomaassociated herpesvirus lytic genes. J. Virol. 84:1334-1347. http://dx.doi .org/10.1128/JVI.01301-09

Portales-Casamar, E., S.Thongjuea, A. T. Kwon, D. Arenillas, X. Zhao, E.Valen, D. Yusuf, B. Lenhard, W.W. Wasserman, and A. Sandelin. 2010. JASPAR 2010: the greatly expanded open-access database of transcription factor binding profiles. Nucleic Acids Res. 38:D105-D110. http://dx.doi.org/ 10.1093/nar/gkp950

Prevorovský, M., T. Grousl, J. Stanurová, J. Rynes, W. Nellen, F. Půta, and P. Folk. 2009. Cbf11 and Cbf12, the fission yeast CSL proteins, play opposing roles in cell adhesion and coordination of cell and nuclear division. Exp. Cell Res. 315:1533-1547. http://dx.doi.org/10.1016/j.yexcr.2008.12.001

Raafat, A., S. Lawson, S. Bargo, M. Klauzinska, L. Strizzi, A.S. Goldhar, K. Buono, D. Salomon, B.K.Vonderhaar, and R. Callahan. 2009. Rbpj conditional knockout reveals distinct functions of Notch4/Int3 in mammary gland development and tumorigenesis. Oncogene. 28:219-230. http://dx.doi.org/10.1038/onc.2008.379

Reedijk, M., S. Odorcic, L. Chang, H. Zhang, N. Miller, D.R. McCready, G. Lockwood, and S.E. Egan. 2005. High-level coexpression of JAG1 and NOTCH1 is observed in human breast cancer and is associated with poor overall survival. Cancer Res. 65:8530-8537. http://dx.doi.org/ 10.1158/0008-5472.CAN-05-1069

Rhodes, D.R., J. Yu, K. Shanker, N. Deshpande, R. Varambally, D. Ghosh, T. Barrette, A. Pandey, and A.M. Chinnaiyan. 2004. ONCOMINE: a cancer microarray database and integrated data-mining platform. Neoplasia. 6:1-6. http://dx.doi.org/10.1016/S1476-5586(04)80047-2

Robertson, G., M. Hirst, M. Bainbridge, M. Bilenky, Y. Zhao, T. Zeng, G. Euskirchen, B. Bernier, R.Varhol, A. Delaney, et al. 2007. Genome-wide profiles of STAT1 DNA association using chromatin immunoprecipitation and massively parallel sequencing. Nat. Methods. 4:651-657. http:// dx.doi.org/10.1038/nmeth1068

Robertson, A.G., M. Bilenky, A. Tam, Y. Zhao, T. Zeng, N. Thiessen, T. Cezard, A.P. Fejes, E.D. Wederell, R. Cullum, et al. 2008. Genome-wide relationship between histone H3 lysine 4 mono- and tri-methylation and transcription factor binding. Genome Res. 18:1906-1917. http://dx.doi.org/10 .1101 /gr.078519.108

Robinson, M.D., D.J. McCarthy, and G.K. Smyth. 2010. edgeR: a Bioconductor package for differential expression analysis of digital gene expression data. Bioinformatics. 26:139-140. http://dx.doi.org/10.1093/bioinformatics/ btp616

Roider, H.G., A. Kanhere, T. Manke, and M.Vingron. 2007. Predicting transcription factor affinities to DNA from a biophysical model. Bioinformatics. 23:134-141. http://dx.doi.org/10.1093/bioinformatics/bt1565

Rubinson, D.A., C.P. Dillon, A.V. Kwiatkowski, C. Sievers, L.Yang, J. Kopinja, D.L. Rooney, M. Zhang, M.M. Ihrig, M.T. McManus, et al. 2003. A lentivirus-based system to functionally silence genes in primary mammalian cells, stem cells and transgenic mice by RNA interference. Nat. Genet. 33:401-406. http://dx.doi.org/10.1038/ng1117

Sahlgren, C., M.V. Gustafsson, S. Jin, L. Poellinger, and U. Lendahl. 2008. Notch signaling mediates hypoxia-induced tumor cell migration and invasion. Proc. Natl. Acad. Sci. USA. 105:6392-6397. http://dx.doi.org/10 .1073 /pnas.0802047105

Vaupel, P. 2004. The role of hypoxia-induced factors in tumor progression. Oncologist. 9:10-17. http://dx.doi.org/10.1634/theoncologist.9-90005-10

Wang, H., J. Zou, B. Zhao, E. Johannsen, T. Ashworth, H. Wong, W.S. Pear, J. Schug, S.C. Blacklow, K.L. Arnett, et al. 2011. Genome-wide analysis reveals conserved and divergent features of Notch1/RBPJ binding in human and murine T-lymphoblastic leukemia cells. Proc. Natl. Acad. Sci. USA. 108:14908-14913. http://dx.doi.org/10.1073/pnas.1109023108

Wingender, E. 2008. The TRANSFAC project as an example of framework technology that supports the analysis of genomic regulation. Brief. Bioinform. 9:326-332. http://dx.doi.org/10.1093/bib/bbn016

Yu, K., K. Ganesan, L.K. Tan, M. Laban, J. Wu, X.D. Zhao, H. Li, C.H. Leung, Y. Zhu, C.L. Wei, et al. 2008. A precisely regulated gene expression cassette potently modulates metastasis and survival in multiple solid cancers. PLoS Genet. 4:e1000129. http://dx.doi.org/10.1371/journal.pgen.1000129

Zambelli, F., G. Pesole, and G. Pavesi. 2009. Pscan: finding over-represented transcription factor binding site motifs in sequences from co-regulated or co-expressed genes. Nucleic Acids Res. 37:W247-W252. http://dx.doi .org/10.1093/nar/gkp464

Zhao, H., A. Langerød, Y. Ji, K.W. Nowels, J.M. Nesland, R. Tibshirani, I.K. Bukholm, R. Kåresen, D. Botstein, A.L. Børresen-Dale, and S.S. Jeffrey. 2004. Different gene expression patterns in invasive lobular and ductal carcinomas of the breast.Mol.Biol. Cell.15:2523-2536.http://dx.doi.org/ 10.1091/mbc.E03-11-0786

Zhou, S., and S.D. Hayward. 2001. Nuclear localization of CBF1 is regulated by interactions with the SMRT corepressor complex. Mol. Cell. Biol. 21 6222-6232. http://dx.doi.org/10.1128/MCB.21.18.6222-6232.2001 